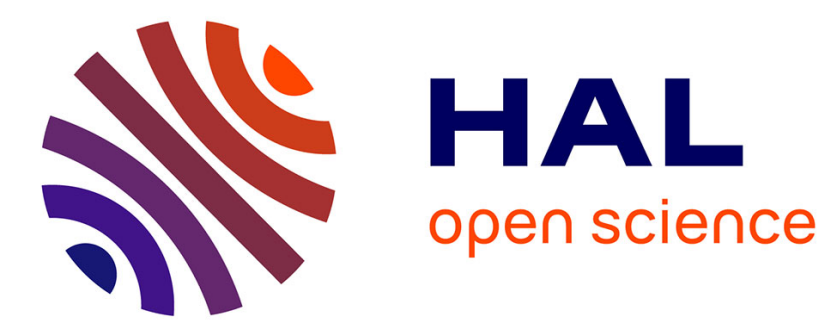

\title{
On multi-carrier systems robustness to Doppler in fast varying flat fading wireless channel
}

\author{
Ahmad Hamdan, Laurent Ros, Hussein Hijazi, Cyrille Siclet, Ali
}

Al-Ghouwayel

\section{- To cite this version:}

Ahmad Hamdan, Laurent Ros, Hussein Hijazi, Cyrille Siclet, Ali Al-Ghouwayel. On multi-carrier systems robustness to Doppler in fast varying flat fading wireless channel. Digital Signal Processing, 2021, 117, pp.103189. 10.1016/j.dsp.2021.103189 . hal-03325410

\section{HAL Id: hal-03325410 \\ https://hal.science/hal-03325410}

Submitted on 24 Aug 2021

HAL is a multi-disciplinary open access archive for the deposit and dissemination of scientific research documents, whether they are published or not. The documents may come from teaching and research institutions in France or abroad, or from public or private research centers.
L'archive ouverte pluridisciplinaire HAL, est destinée au dépôt et à la diffusion de documents scientifiques de niveau recherche, publiés ou non, émanant des établissements d'enseignement et de recherche français ou étrangers, des laboratoires publics ou privés. 


\title{
On Multi-carrier Systems Robustness to Doppler in Fast Varying Flat Fading Wireless Channel ${ }^{\star}$
}

\author{
Ahmad HAMDAN, Laurent ROS, Hussein HIJAZI, Cyrille Siclet and Ali AL-GHOUWAYEL
}

ARTICLE INFO

\section{Keywords:}

OFDM

CP-OFDM

FBMC

Doppler

Rayleigh

\begin{abstract}
A B S TRACT
In this paper, the performance of a generalized multi-carrier Frequency Division Multiplexing (FDM) system - including Orthogonal Frequency Division Multiplexing (OFDM) and Filter-Bank MultiCarrier (FBMC) - is analyzed in terms of normalized interference power in a fast-varying flat-fading downlink channel. The analysis is done assuming a conventional correlator-based receiver using three families of pulse shapes: orthogonal time-limited pulse shapes, orthogonal frequency-limited pulse shapes, and non-orthogonal pulse shapes. The analysis is done for the Rayleigh random channel with different Doppler spreads, and interference distribution over sub-carriers and symbols is analyzed per pulse shape. Interference reduction by reducing the bandwidth efficiency through turning off some subcarriers and reducing symbol density is then analyzed. After that, the special ability of non-orthogonal pulse shapes to have more flexible density reduction is indexed and studied. The theoretical study, validated by Monte Carlo simulations, showed that for low, high, and extreme Doppler spreads, the appropriate pulse shapes (resp. system density) to be used are respectively time or frequency limited orthogonal pulse shapes (resp. 100\%), short-tail frequency-limited pulse shapes (resp. 50\%), and time and frequency limited non-orthogonal pulse shapes (resp. 25\%).
\end{abstract}

\section{Introduction}

Communication systems utilizing Multi-Carrier (MC) technologies are known for their robustness over multi-path channels and are used in many scenarios requiring multiplexing and/or flexibility. Regarding the multi-path scenarios, it is well known that using a single-carrier system with a short pulse duration for high symbol-rate transmission over timedispersive channels makes the system vulnerable to InterSymbol Interference (ISI). Hence, by splitting the wide bandwidth (high symbol-rate) data stream into narrower sub-bands each modulated over a different sub-carrier, MC transmission can significantly reduce the impact of multi-path (like ISI and frequency selectivity) which decreases with the increase of symbol duration [1]. MC transmissions are also used for multiple access [2], broadcasting [3], satellite communications [4], and terahertz communications [5] through scenarios where a single path channel can be assumed. For example in terahertz communications, $\mathrm{MC}$ is used mainly for its parallelization feature to avoid implementational issues.

On the other hand, due to their reduced bandwidth (per sub-carrier), MC transmissions may be more vulnerable to Inter-Carrier Interference (ICI). Such impairment occurs when the channel exhibits time-variations within one $\mathrm{MC}$ symbol, i.e. in presence of a time-selective (frequencydispersive, frequency-shifting,...) channel. This can occur because of Radio-Frequency imperfections like carrier frequency offset [6], or phase noise instabilities [7], which is a big issue for terahertz oscillators [8]. However, the more

${ }^{\star}$ This work has been partially supported by the LabEx PERSYVAL-Lab (ANR-11-LABX-0025-01) funded by the French program Investissement d'avenir.

@ ahmad.hamdan@univ-grenoble-alpes.fr (A. HAMDAN);

laurent. ros@gipsa-lab.grenoble-inp. fr (L. ROS);

hussein. hijazi@liu. edu.lb (H. HIJAZI);

cyrille.siclet@gipsa-lab.grenoble-inp. fr (C. Siclet);

ali.ghouwayel@efrei.fr (A. AL-GHOUWAYEL) direct physical reason that induces ICI in MC systems is the Doppler effect $[9,10]$ caused by the mobility of the receiver with respect to the transmitter, which is the situation addressed in this paper. More specifically, we assume a fixed transmitter (e.g. base station) and a mobile receiver with uniform velocity in a scattering environment (e.g. in a car). In such a scenario, the Doppler effect does not result in a simple deterministic frequency shift but in a more complex random channel with a Doppler spectrum and signal fading distribution [11].

Although the level of interference depends directly on the value of the Doppler spread, it also depends on the parameters of the MC systems, especially on the waveforms used and their localization in time and frequency domains. The Rectangular (Rect) pulse adopted by Orthogonal Frequency Division Multiplexing (OFDM) is well localized in time, but it can induce high ICI in frequency-dispersive channels. This vulnerability is due to its significant overlap with the other carriers in the frequency domain. The use of different pulse shapes in Filter-Bank Multi-Carrier (FBMC) allows selecting pulses that might be more robust against frequency-dispersive channels [12]. A more flexible design can be obtained by using Bi-orthogonal Frequency Division Multiplexing (BFDM) which allows pulse shapes to be different at the transmitter and the receiver [13]. BFDM has the flexibility to be configured such that it is equivalent to FBMC by using matched pulses at the transmitter and the receiver. So, even if the previous works provided some partial view on the robustness of some waveforms, a deeper theoretical analysis focusing on the impact of Doppler spread on both ICI and ISI problems, especially for high Doppler spreads, is still missing. The paper aims is to fill that gap, with some innovations as described below. The authors in [14] have provided theoretical and detailed analysis for FBMC in terms of interference level. Some other similar works were provided for BFDM $[15,16]$. 
However, for all the mentioned works, high values of the normalized Doppler spread (which would apply for systems considering high carrier frequencies) were not considered. In this work, deeper analysis and interpretation of the fading effects especially the ISI for high Doppler spreads, interference localization, and flexible density reduction, are provided. This leads to a more advanced discussion in terms of interference properties. In addition, new theoretical equations formulating the interference level for a given Doppler spread and given system parameters are presented. These equations permit to provide detailed analysis at separate ISI and ICI levels versus offset for such systems. Another contribution is the approach considered for the interference reduction by using fewer resources, while the required reduction ratio is analyzed and the appropriate pulse shape for each range of Doppler spread is decided. Finally, a soft spacing approach with a relative theoretical analysis is proposed.

This work is done following the assumption of single-path fast-varying channels. Such a single-path channel model is a good approximation for scenarios having the coherence bandwidth $B_{\mathrm{Coh}}=1 / D$ of the channel (due to a delay spread $D$ ) much greater than the signal's bandwidth $B$, whatever the variation speed (slow or fast) of the channel is. The system is assumed to use a conventional correlator-based receiver without considering equalization or interference cancellation. This setup was assumed to measure the inner robustness of the waveforms, regardless of any advanced or complex signal processing techniques, conversely to our previous studies [17]. To sum up, the contributions are as follows:

1. describing a new analysis and preview of the problem of Doppler spread and its impact on time-limited and frequency-limited pulse shapes,

2. analyzing the behavior of orthogonal pulse shapes overlapping in time in presence of high Doppler spread leading to the problem of Doppler-generated-ISI,

3. providing a generalized system model that is valid for a single path propagation multi-channel communication which was later narrowed to multi-carrier, and deriving the theoretical expected received power at each timefrequency slot generated due to the transmission of a specific time-frequency slot,

4. analyzing the over-sampled multi-carrier systems' performance in fast-varying channels using the derived theoretical expected values of ISI and ICI versus normalized Doppler spread assuming Rayleigh channel with Jakes's Doppler spectrum (Clarke model [11, 18]) for different families of pulse shapes,

5. analyzing the time-frequency localization of interference (ISI and ICI) by using the derived theoretical expression versus the offset relative to the index of the desired time-frequency slot,

6. discussing the need of reducing the time-frequency grid density in fast-varying channels to obtain a lower level of interference and a more usable channel,

7. discussing the special ability of non-orthogonal pulse shapes to have an arbitrary offset between symbols/subcarrier without requiring alignment,

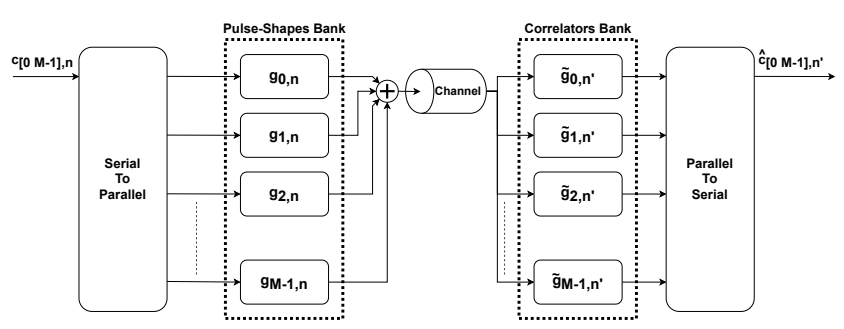

Figure 1: MC System Schematic.

8. providing Monte-Carlo simulation results that validate all the theoretical interference formulas.

The rest of this paper is organized as follows: section 2 provides a brief review of MC systems, motivation explaining disturbances caused by Doppler spread. This was done by providing a simplified system model along with understandable illustrated examples. Theoretical interference analysis and related derivations are provided in section 3. Simulation results are displayed in section 4 along with Monte-Carlo results confirming the theoretical derivations. Analysis based on the results is discussed in the same section including timefrequency grid density reduction. Based on the density reduction analysis, the special ability of non-orthogonal pulse shapes of having arbitrary spacing in time and frequency is indexed in section 5, where theoretical interference is recalculated based on this property, and supported by Monte-Carlo results. Finally, the paper is concluded in section 6 .

\section{Preliminaries and Motivations}

This section reviews the most important MC-based systems, gives the preliminaries and motivations of this work, and presents our work's system model.

\subsection{Multi-carrier Systems Review}

Multi-carrier transmission is transmitting a stream of encoded data by multiplexing it over multiple carrier frequencies each defined by transmitting and receiving pulse shapes (or waveforms) as shown in Figure 1. The aim is to minimize the cross-talk between carriers and consecutive symbols by trying to achieve the relation:

$$
v_{m, n, m^{\prime}, n^{\prime}}=\left\langle\boldsymbol{g}_{m, n}, \tilde{\boldsymbol{g}}_{m^{\prime}, n^{\prime}}\right\rangle \approx \begin{cases}\text { Const, } & m=m^{\prime} \\ 0, & \text { otherwise }\end{cases}
$$

where:

- $\langle\boldsymbol{x}, \boldsymbol{y}\rangle$ is the inner product between $\boldsymbol{x}$ and $\boldsymbol{y}$ such that $\langle\boldsymbol{x}, \boldsymbol{y}\rangle=\sum_{q} x[q] y^{*}[q]$.

- $\boldsymbol{g}_{m, n}$ is the transmit pulse shape of the $m^{\text {th }}$ sub-carrier of the $n^{\text {th }}$ FDM transmitted symbol.

- $\tilde{\boldsymbol{g}}_{m^{\prime}, n^{\prime}}$ is the receiver pulse shape used to recover (by correlation) the $m^{\prime t h}$ sub-carrier of the $n^{\prime t h}$ FDM received symbol. In the case of a conventional matched filter receiver, $\tilde{\boldsymbol{g}}_{m^{\prime}, n^{\prime}}=\boldsymbol{g}_{m^{\prime}, n^{\prime}}$. 
It is to note that Figure 1 is the scheme of a more general multichannel linear system [19], that can be represented in terms of the transmit pulse shapes and the receive pulse shapes followed by symbol-time synchronous sampling. Then, $\boldsymbol{g}_{m, n}[q]$ can also be regarded as the impulse response of the transmit pulse shape for the channel $m$, and $\tilde{\boldsymbol{g}}_{m^{\prime}, n^{\prime}}^{*}[-q]$ as the impulse response of a receiver pulse shape operating before symboltime sampling for channel $\mathrm{m}^{\prime}$. This scheme stands for any multi-channel linear systems, as for Code Division Multiple Access (CDMA) for which the waveforms may belong to different users and be built from orthogonal codes [20,21]. Moreover, Equation 1 can be regarded as a generalization of the interference-free Nyquist criterion [22], extended to the multi-channel systems. Further, in the case of a time-varying single path channel with a complex gain $h[q]$, the transmit pulse $\boldsymbol{g}_{m, n}$ should be replaced by $\boldsymbol{h} . \boldsymbol{g}_{m, n}$ in Equation 1. Introducing also the index $\Delta=\left[\Delta_{c}, \Delta_{s}\right]$ having its components: sub-carrier offset $\Delta_{c}$, and symbol offset $\Delta_{s}$ :

$$
\begin{aligned}
\Delta & =\left[\Delta_{c}, \Delta_{s}\right] \\
\Delta_{c} & =m^{\prime}-m \\
\Delta_{s} & =n^{\prime}-n,
\end{aligned}
$$

Equation 1 can be rewritten as:

$$
v_{\Delta}=\left\langle\boldsymbol{h} \cdot \boldsymbol{g}_{m, n}, \tilde{\boldsymbol{g}}_{m^{\prime}, n^{\prime}}\right\rangle \approx \begin{cases}\text { Const, } & \Delta=\left[\begin{array}{ll}
0 & 0
\end{array}\right] \\
0, & \text { otherwise. }\end{cases}
$$

A consequence of Equation 3 implies that the bandwidth $\boldsymbol{B}_{s c}$ of a sub-channel (a sub-carrier in the case of MC system) should be greater or equal to $1 / T_{S}$ for an MC symbol time $T_{S}$ :

$$
B_{s c} \geq B_{s c}^{\min }=1 / T_{S} \text {. }
$$

This minimum bandwidth is a necessary (but non-sufficient) condition to make the interference-free condition possible in the case of a static single-path channel.

In classical multi-carrier transmission systems, parallel data streams are transmitted over multiple non-overlapping frequency sub-channels each having a separate data symbol stream to be modulated with. The used sub-channels are usually separated with guard bands $G$ to avoid ICI which reduces the spectral efficiency. Such systems are usually known as Frequency Division Multiplexing (FDM) systems where some bandwidth $B$ is divided into $M$ sub-bands/subchannels each of bandwidth $B_{s c}=B / M-G$ and symbols are multiplexed over them. Classical FDM is known for its reduced bandwidth efficiency compared to Single Carrier (SC) transmission due to the guard bands required to reduce ICI. However, it is known for its robustness against impairments resulted from multi-path channels due to the increased symbol duration $T_{S}$ caused by decreased sub-band bandwidth. On the other hand, FDM is known for its improved time efficiency where the symbols are overlapped in time so that they do not require the guard-time intervals as in SC [23]. SC can be viewed as Time Division Multiplexing (TDM) where the input stream is modulated over consecutive time slots. As discussed in [24], FDM and SC / TDM have equivalent total efficiency where: what FDM gains in time, loses it in frequency, and vice versa.

More advanced variants of FDM are OFDM and FBMC. OFDM [25] is widely used due to its increased spectral efficiency compared to the classical multi-carrier systems, its ability to be easily implemented using Discrete Fourier Transform (DFT) [26], and can be easily extended to CP-OFDM having multiple benefits [27]. FBMC was first introduced in the same work with OFDM [25], but with the ability to use some pulse shape other than the Rect pulse used in OFDM. FBMC has grabbed more interest with concatenation Offset Quadrature Amplitude Modulation (OQAM) in Filter-Bank Multi-Carrier with Offset Quadrature Amplitude Modulation (FBMC-OQAM) [28]. Another variant of FDM is BFDM [13], which allows using transmitting and receiving pulses that are not matched but yet bi-orthogonal. This can allow relaxing the design of the pulses to serve some target other than maintaining the best SNR as in [16]. For the mentioned FDM variants, the sample $q$ of the pulse $g_{m, n}$ (and similarly for $\tilde{g}_{m, n}$ ) defined in Equation 1 can be represented as:

$$
g_{m, n}[q]=g\left(q T_{S a}-n T_{S}\right) e^{j 2 \pi m F_{S}\left(q T_{S a}-n T_{S}\right)}
$$

such that $g(t)$ is the prototype pulse value at instant $t, T_{S a}$ is the sample time, and $F_{S}$ is the sub-carrier spacing. The variation of $\boldsymbol{h}$ might result in having $\left|v_{\Delta}\right|>0$ for $\Delta \neq\left[\begin{array}{ll}0 & 0\end{array}\right]$ (that means Equation 3 might be not satisfied even if Equation 1 is satisfied). This effect is discussed in detail in the next section.

\subsection{Doppler Spread Impact On Multi-Carrier Transmission}

\subsubsection{Communication Channel Variation and System's Parameters}

For MC transmissions, since data are multiplexed over several sub-carriers, these sub-carriers should have a minimum correlation. For this reason, first FDM techniques used pulse shaping with reduced sub-carrier bandwidth and a guard band between sub-carriers, while more advanced techniques like OFDM, FBMC, and BFDM reduced this correlation to zero by designing orthogonal waveforms. As seen in Figure 2, a static (or slow fading) channel having no (or negligible) time-variation, will have no (or negligible) spreading in the Doppler frequency domain. Then, static or slow fading channels -if no other impairments are considered- will maintain the properties of designed pulse shapes in terms of interference avoidance. However, for both scenarios: basic and advanced techniques, a fast-moving transceiver will suffer from a fast-varying channel leading to a Doppler frequency spreading behavior as illustrated in Figure 3. Such Doppler frequency spreading channel will break the orthogonality and alter the time-frequency localization for orthogonal techniques, and expand wider than the guard-band if it is not sufficient for classical FDM techniques; consequently introducing ICI in both cases.

To be more accurate, to state if a communication chan$n e l$ is a slow or fast fading channel, we have so compare the 

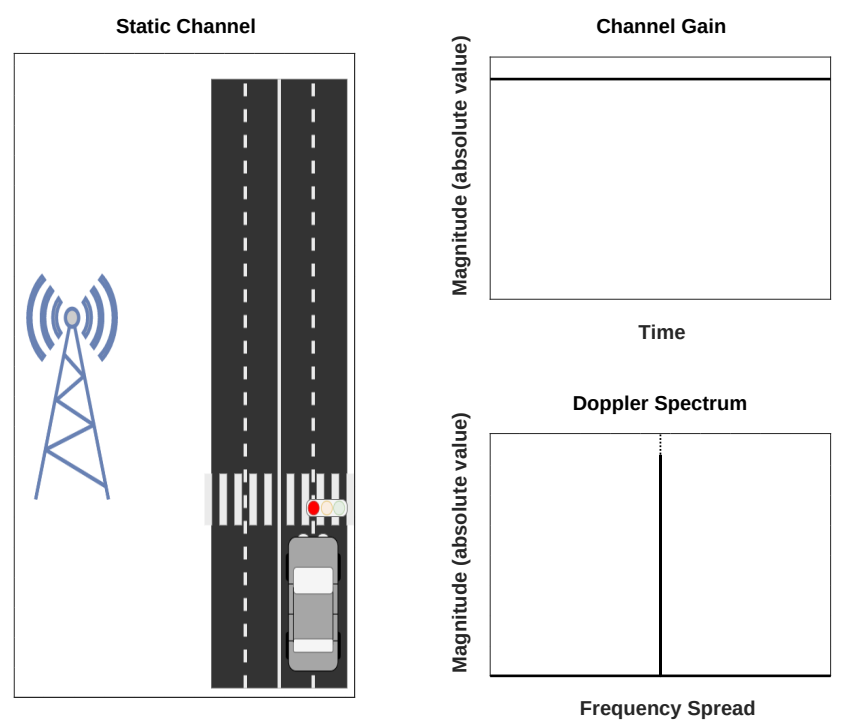

Figure 2: Slow-varying channel illustration.

dynamic parameters of the propagation channel to those of the communication system. A communication channel will be considered as slow-fading if it has a negligible variation within one MC symbol period $T_{S}$ expressed as $T_{\mathrm{Coh}} \gg T_{S}$, where $T_{\mathrm{Coh}}$ is the coherence time of the channel, equals to the reciprocal of the Doppler frequency, $T_{\mathrm{Coh}}=1 / F_{d}$. Equivalently, it means that the Doppler spread $F_{d}$ should be negligible compared to the symbol rate $D_{S}=1 / T_{S}$, i.e. $F_{d} \ll D_{S}$. In other words, the speed of a communication channel can be stated by the value of the normalized Doppler frequency $F_{d} T_{S}$, where $T_{S}$ is the MC symbol time, and $F_{d}$ the Doppler frequency. Using the $F_{d} T_{S}$ metric just mentioned, a communication channel is considered to be slow-varying if $F_{d} T_{S} \ll 1$, conventionally recognized as $F_{d} T_{S} \leq 10^{-2}$ or $10^{-3}$. On the contrary, the fast varying communication channel corresponds to an increase in $F_{d}$ and then a decrease of $T_{\mathrm{Coh}}$ such that $T_{\mathrm{Coh}} \rightarrow T_{S}$ so that $F_{d} \cdot T_{S} \rightarrow 1$. A communication channel is conventionally recognized to be fast if $F_{d} \cdot T_{S} \geq 0.1$. Notice that for a channel to be invariant for some pulse of duration $K T_{S}$ and maintain pulses' orthogonality, the variation should be negligible over the whole pulse duration $K T_{S}$ not just over $T_{S}$. However, the study will consider the input parameter $F_{d} T_{S}$ to compare the pulses to get a fair comparison for a given Doppler Spread and a given MC symbol rate $1 / T_{S}$, even if the pulses have different duration.

\subsubsection{Doppler-generated-ICI}

A more detailed illustration of the impact of Doppler spread in the frequency domain on the most popular timelimited pulse shape (Rect) and the most popular frequencylimited pulse shape (RRC) is shown in Figure 4 and Figure 5 respectively.

In these figures, the first column represents the static channel ideal scenario (without Doppler) or slow-varying channel. The second column represents the scenario with a fast-varying channel (strong variations within one $T_{S}$, such that $F_{d} T_{S}=0.2$ ). The frequency-domain magnitude of one
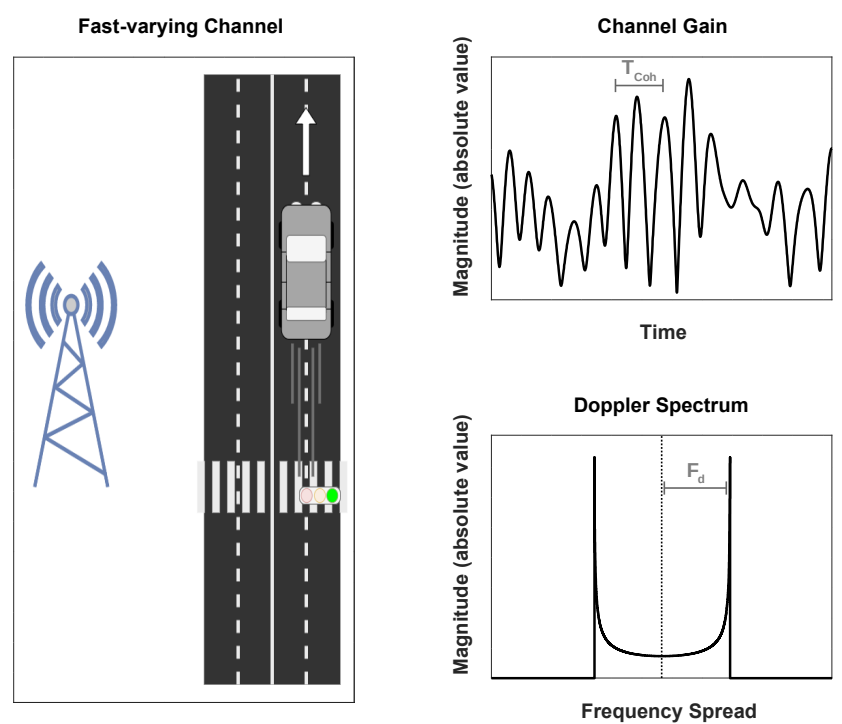

Figure 3: Fast varying channel illustration.

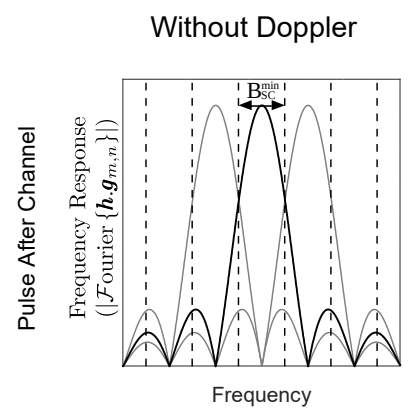

With Doppler ( $\left.{ }_{\mathrm{d}^{\top} \mathrm{s}}=0.2\right)$
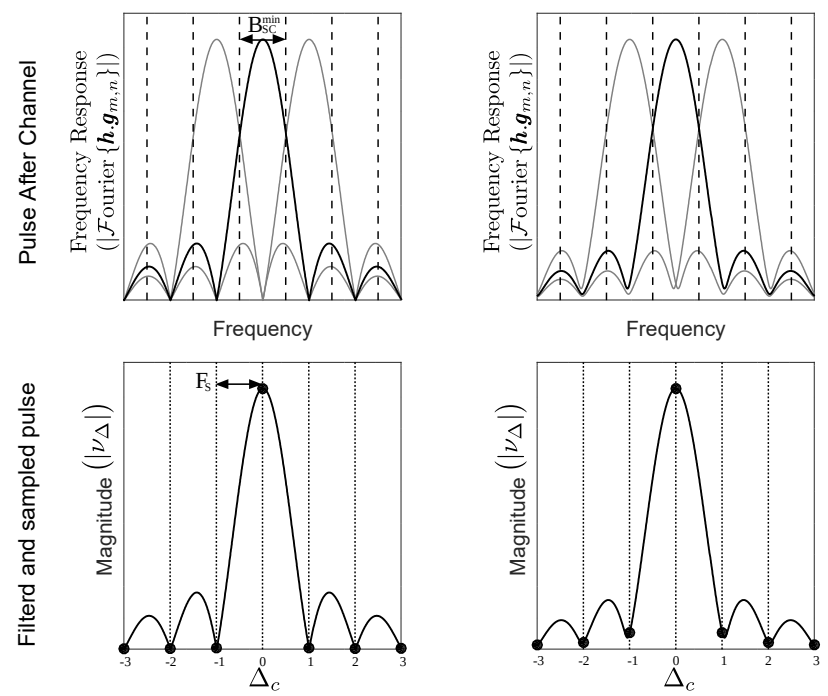

Frequency

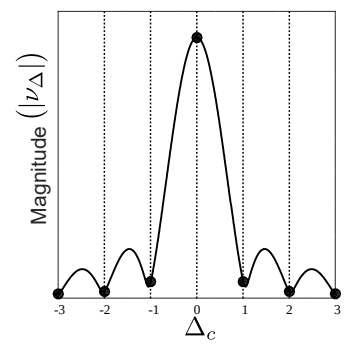

Figure 4: Rect Pulse - Doppler Spread Frequency Impact.

given sub-carrier pulse after the channel is shown in the first row, with also adjacent sub-carriers in a shade of gray, and the space between two vertical dashed lines delimits the minimal bandwidth, $B_{s c}^{\min }=1 / T_{S}$, for every sub-carrier. It is worth mentioning that for the scenarios analyzed in Figure 4 and Figure 5, the system is configured such that the space between sub-carriers, $F_{S}=1 / T_{S}$, corresponds to $B_{s c}^{\min }$, i.e. $F_{S}=$ $B_{s c}^{\min }$. In the second row, the magnitude response to one given transmit (TX) sub-carrier pulse $\left(\boldsymbol{g}_{m, n}\right)$ is computed after being subjected to channel's (possibly variable) gains $\left(\boldsymbol{h} \cdot \boldsymbol{g}_{m, n}\right)$, then matched correlation with RX pulses with arbitrary RX Carrier Frequencies $\left(\Delta_{c} \in \mathbb{R}\right)$. The frequency of each $\mathrm{RX}$ sub-carrier $\left(\Delta_{c} \in \mathbb{Z}\right)$ is shown by vertical dotted lines and the magnitude calculated at each sub-carrier is shown by a black-filled circle.

For the analysis of the impact of Doppler spread on Rect 


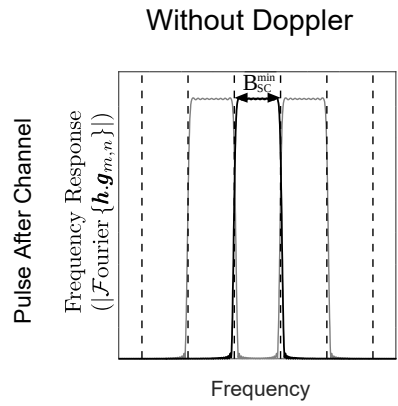

$$
\text { With Doppler }\left(\mathrm{F}_{\mathrm{d}} \mathrm{T}_{\mathrm{s}}=0.2\right)
$$
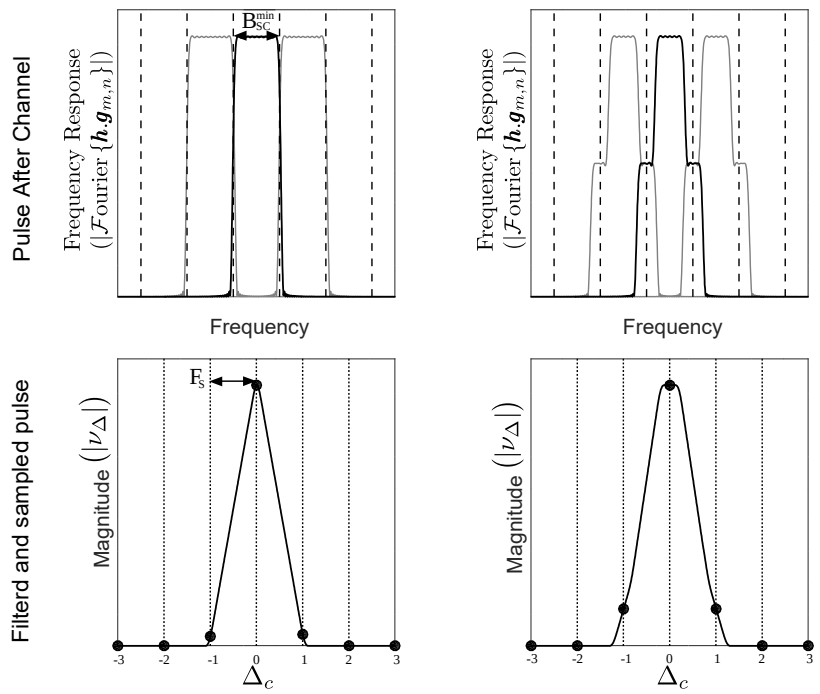

Figure 5: RRC Pulse - Doppler Spread Frequency Impact.

pulse shown in Figure 4, in a static channel, the symbol pulse is similar at transmitter output and receiver input since the symbol is multiplied by a constant. Consequently, The frequency-domain magnitude of the pulse is not altered, and the magnitude at each sub-carrier position after receiving correlation is zero except for the center desired frequency since the pulse is perfectly aligned although not well localized $\left(\left|v_{\Delta}\right|=0\right.$ for $\left.\Delta_{c} \in \mathbb{Z}^{*}\right)$. However, it can be noticed that any offset (or synchronization error) in the sub-carriers grid position or Doppler spreading in the channel response will lead to severe interference at all sub-carriers since the value is rapidly increasing after getting zero at the exact sub-carrier frequency. For the time-varying channel, the symbol pulse at the receiving correlator input is different from the one at the output of the transmitting pulse shape due to multiplication by a variable channel gain within one symbol period $T_{S}$. Consequently, the pulse frequency is spread in the Doppler frequency domain. This change in the frequency response of the pulse, leads to a different response after the correlation at the receiver, resulting in non-zero values even at $\mathrm{RX}$ sub-carriers positions different than the center desired TX position, leading to what can be called Doppler-generated-ICI by having $\left|v_{\Delta}\right|>0$ for one or more values $\Delta_{c} \in \mathbb{Z}^{*}$.

For the analysis of the impact of Doppler spread on RRC pulse shown in Figure 5, even in an ideal static channel, slight ICI is generated. This is due to 0.1 roll-off parameter of the RRC pulse shape used ( 0 roll-of cannot be realized in practice due to infinite length impulse response which is hard to be truncated because of strong sinc function side lobes), since we assume here a minimum subcarrier spacing $\left(F_{S}=1 / T_{S}\right)$. Note that the sub-carrier spacing may however be greater in the general model of subsection 2.3. In a fast varying channel, the spreading in frequency increased the interference. However, it is still limited to the first adjacent neighbors in contrary to time-limited pulse shapes.

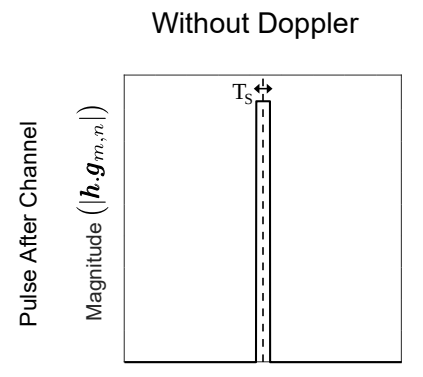

With Doppler $\left(\mathrm{F}_{\mathrm{d}} \mathrm{T}_{\mathrm{s}}=0.2\right)$
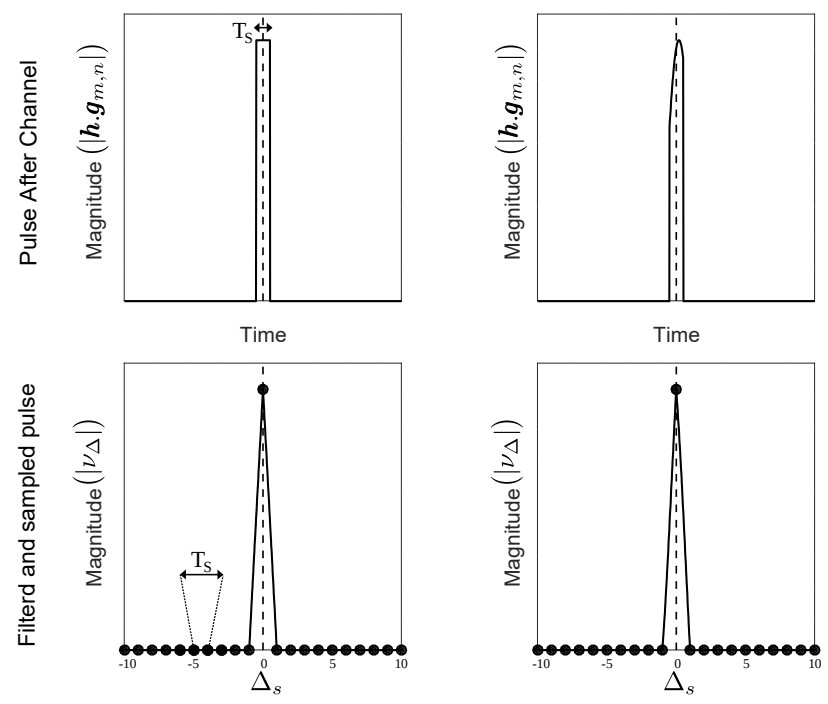

Figure 6: Rect Pulse - Doppler Spread Time Impact.

\subsubsection{Doppler-generated-ISI}

Another interesting impact of the Doppler effect in the time domain, already occurring for single carrier and single pulse, is illustrated in Figure 6 and Figure 7 for the Rect pulse shape and the RRC pulse shape respectively. In these figures, the first column represents the ideal scenario of an ideal static channel (without Doppler), and the second column represents the scenario with a fast-varying channel (with strong normalized Doppler). The $0 \mathrm{~Hz}$ sub-carrier pulse after channel (corresponding to $\boldsymbol{h} \cdot \boldsymbol{g}_{0, n}$ ) is shown in the first row and will be next considered as unique input for the receiver (in the input of $\tilde{g}_{0, n^{\prime}}$ in Figure 1). In the second row, we show then the magnitude response to the previous pulse after correlation, having the RX carrier frequency equaling the desired TX frequency $\left(\Delta_{c}=0\right)$, but computed for different time offsets.

The magnitude is calculated at each arbitrary time-offset $\Delta_{s} \in \mathbb{R}$, and discrete symbol time offsets such that $\Delta_{s} \in \mathbb{Z}$ are shown by the black-filled circles. Due to having the timelimited pulse of Rect pulse shape as shown in Figure 6, in a single path fast varying channel, the values of the pulse will vary without introducing any interference power to the adjacent symbols. Consequently, the magnitude calculated in the second row is maximum at the desired symbol offset and zero otherwise for both scenarios with no Doppler and with high Doppler $\left(\left|v_{\Delta}\right|=0\right.$ for $\left.\Delta_{s} \in \mathbb{Z}^{*}\right)$.

Furthermore, for a frequency-limited/time-spreading pulse like the RRC pulse shown in Figure 7, in a single path static channel, although the pulse is spreading in the time domain, it still maintains the orthogonality where the inner product at offsets of integer multiples of a symbol period is zero except at the desired symbol $\left(\left|v_{\Delta}\right|=0\right.$ for $\left.\Delta_{s} \in \mathbb{Z}^{*}\right)$. It means that the ISI-free Nyquist Criterion [22] is satisfied. On the other hand, for fast varying channels, the orthogonality is broken due to the altering of the shape of the spreading pulse 


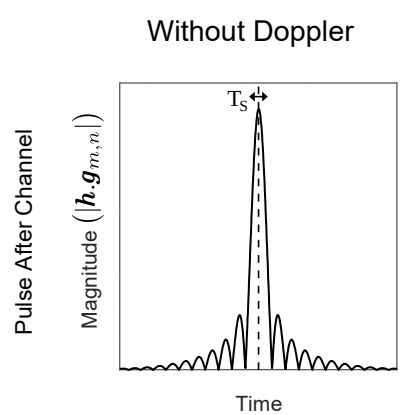

With Doppler $\left(\mathrm{F}_{\mathrm{d}} \mathrm{T}_{\mathrm{s}}=0.2\right)$
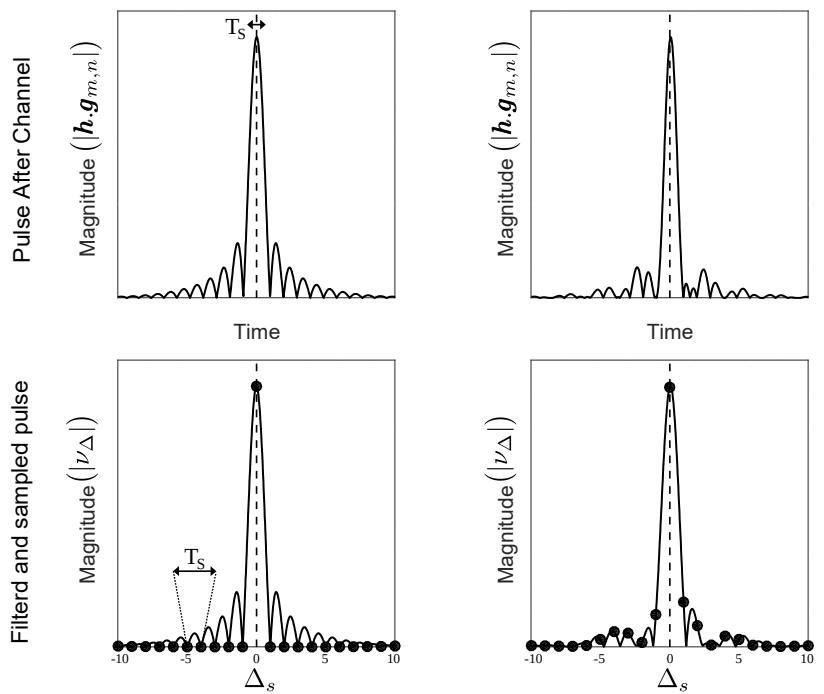

Figure 7: RRC Pulse - Doppler Spread Time Impact.

and consequently changing the results of the inner-products to have $\left|v_{\Delta}\right|>0$ for some $\Delta_{s} \in \mathbb{Z}^{*}$ what can be called Doppler-generated-ISI.

Based on the above discussion, it is interesting to look into how would the interference vary based on the selected pulse shape, and normalized Doppler spread $\left(F_{d} T_{S}\right.$ for Doppler frequency $F_{d}$ and MC symbol time $T_{S}$ ). Furthermore, it is interesting to check how the interference is spread over neighbor frequency sub-carriers and adjacent time symbols. The answers to these questionings will be given in this paper.

\subsection{System Model}

\subsubsection{Assumptions}

In this paper, the following assumptions will be used:

- Noise is negligible, or not considered, to focus only on interference impact.

- Input symbols $c_{m, n}$ are Independent and Identically Distributed (i.i.d). They are also assumed zero-mean, and complex values, as typically Quadrature-Amplitude Modulation (QAM) symbols.

- Single path channel.

- Channel is Wide Sense Stationary (WSS).

\subsubsection{Notations}

In this paper, the following notations will be used:

- $s_{\mathrm{sub}_{1}, \mathrm{sub}_{2}, \ldots}$ is the part of the signal/set $s$ that is related to the properties $\operatorname{sub}_{1}$, $\operatorname{sub}_{2}, \ldots$ For example, $s_{m, n}$ can represent the signal $s$ that is generated for sub-carrier $m$ and symbol $n$.

- $s(t)$ is the sample of signal $s$ at instance $t$.

- $s$ is the vector of samples of $s(t)$ sampled at $t=k T_{S a}$ for $k \in \mathbb{Z}$, where $T_{S a}$ is the sampling period.
- $s[k]$ is the $k^{t h}$ sample of the discrete vector $\boldsymbol{s}$.

In the next section, the system model for multi-channel systems is presented, then narrowed to a model of multi-carrier systems.

\subsubsection{Multi-channel Model}

Let $N$ be the number of samples spacing between two consecutive multi-channel symbols such that for a sample duration $T_{S a}$ the multi-channel symbol (interval) duration is $T_{S}=N T_{S a}, K$ be the time span of the pulse in terms of multi-channel symbols (number of pulse samples $/ N$ ) such that $K N$ is an integer, and equivalently $K-1$ be the overlapping factor. The $q^{\text {th }}$ sample of $n^{\text {th }}$ multi-channel symbol over the $m^{\text {th }}$ sub-channel for the input symbol $c_{m, n}$ is written as:

$$
s_{m, n}[q]=c_{m, n} g_{m, n}[q] \quad q \in[n N ;(K+n) N-1] .
$$

Let $M$ be the number of sub-channels. Summing over all sub-channels, the $q^{\text {th }}$ sample of $n^{\text {th }}$ multi-channel symbol for the input symbols $c_{m, n}$ will be:

$$
\begin{aligned}
s_{n}[q] & =\sum_{m} s_{m, n}[q] \\
& =\sum_{m} c_{m, n} g_{m, n}[q] \quad q \in[n N ;(K+n) N-1],
\end{aligned}
$$

The signal at the input of the receiver will then be equal to:

$$
r[q]=(\boldsymbol{h} . \boldsymbol{s})[q]=h[q] s[q],
$$

such that:

- $s[q]=\sum_{n} s_{n}[q]$

- $r[q]$ is the $q^{\text {th }}$ sample of the received multi-channel symbol stream $\boldsymbol{r}$.

- $h[q]$ is the $q^{\text {th }}$ sample of the single discrete timevarying channel $\boldsymbol{h}$.

- . is the Hadamard Product operator.

Using operations matched to those at the transmitter, received symbol at $m^{\prime t h}$ sub-channel, ${n^{\prime}}^{t h}$ symbol $c_{m^{\prime}, n^{\prime}}$ can be expressed as:

$$
\hat{c}_{m^{\prime}, n^{\prime}}=\left\langle\boldsymbol{r}, \tilde{\boldsymbol{g}}_{m^{\prime}, n^{\prime}}\right\rangle=\sum_{q} \tilde{g}_{m^{\prime}, n^{\prime}}^{*}[q] r[q],
$$

matching the flow in Figure 1 such that the design of $g_{m, n}$ and $\tilde{g}_{m^{\prime}, n^{\prime}}$ aims to achieve the relation in Equation 1.

\subsubsection{Multi-carrier Model}

Defining the channels' nature of a multi-channel system is done by defining the transmitting and receiving pulse shapes. In this work, since multi-carrier systems are targeted, transmitting and receiving pulse shapes are defined to operate for sub-carriers. Thus, having the transmitting pulse shape as:

$$
g_{m, n}[q]=g[q-n N] e^{j 2 \pi \frac{m(q-n N)}{M}},
$$


with $q \in[n N ;(K+n) N-1]$ and where $g[q]$ is the $q^{t h}$ sample of the transmitting prototype pulse. Notice that the sub-carrier spacing is just $\frac{1}{M}$ in the discrete representation (instead of $F_{S}=\frac{B}{M}=\frac{N}{M T_{S}}$ in the analog one), which is equivalent to Equation 4 with setting $F_{S}=\frac{N}{M T_{S}}$ and $T_{S}=N T_{S a}$. Using Equation 6 and Equation 9 the sample stream of the $n^{\text {th }}$ transmitted symbol will be:

$$
s_{n}[q]=\sum_{m} c_{m, n} g[q-n N] e^{j 2 \pi \frac{m(q-n N)}{M}},
$$

and the sample stream of all the transmitted symbols summed over $n$ will be:

$$
s[q]=\sum_{n} \sum_{m} c_{m, n} g[q-n N] e^{j 2 \pi \frac{m(q-n N)}{M}} .
$$

The receiving pulse is given by:

$$
\tilde{g}_{m^{\prime}, n^{\prime}}[q]=\tilde{g}\left[q-n^{\prime} N\right] e^{j 2 \pi \frac{m^{\prime}\left(q-n^{\prime} N\right)}{M}},
$$

with $q \in[n N ;(K+n) N-1]$ and where $\tilde{g}[q]$ is the $q^{\text {th }}$ sample of the receiving prototype pulse. It is worthwhile mentioning that we use the same (or matched, orthogonal) pulse shapes at the transmitter and the receiver in our simulations in section 4, even if the derivations are handled, for the sake of generalization, with possibly different pulse shapes, as for BFDM. Using Equation 8 and Equation 12:

$$
\begin{aligned}
\hat{c}_{m^{\prime}, n^{\prime}} & =\left\langle\boldsymbol{r}, \tilde{\boldsymbol{g}}_{m^{\prime}, n^{\prime}}\right\rangle \\
& =\sum_{q} \tilde{g}^{*}\left[q-n^{\prime} N\right] r[q] e^{-j 2 \pi \frac{m^{\prime}\left(q-n^{\prime} N\right)}{M}} .
\end{aligned}
$$

This equation implies that the transmitting and receiving pulses overlap if $g[q-n N] \tilde{g}^{*}\left[q-n^{\prime} N\right] \neq 0$. Hence, the transmitting and receiving prototype filters $g[q]$ and $\tilde{g}[q]$ are defined for $0 \leq q \leq K N-1$ (in other words they are zero otherwise). Therefore, the transmitting and receiving pulses overlap if

$$
\left\{\begin{array}{l}
n N \leq q \leq \quad q \leq(K+n) N-1 \\
n N \leq q-n^{\prime} N \leq(K+n) N-1
\end{array}\right.
$$

which means that

$$
\left(n+n^{\prime}\right) N \leq q \leq(K+n) N-1
$$

assuming $n, n^{\prime} \geq 0$.

\subsubsection{Interference Definition}

In multi-carrier systems, interference can be defined - as seen before - by two terms ICI and ISI. Ideally, the total power received should be equal to the desired power (equivalently meaning that the desired components in output of the bank of RX pulse shapes hold all the output power) such that:

$$
\text { Power }_{\text {Received }}=\text { Power }_{\text {Desired }}=\text { Power }\left.\right|_{m^{\prime}=m, n^{\prime}=n} \text {. }
$$

However, due to some channel or pulse shape imperfections, it is possible to have

$$
\text { Power }_{\text {Received }} \neq \text { Power }_{\text {Desired }} \text {. }
$$

ICI is an impairment in multi-carrier systems caused by the adjacent sub-carriers mainly due to Doppler spread. ICI is a form of distortion of a signal in which one symbol interferes with symbols of the adjacent sub-carriers. In this paper, ICI will be referencing the ratio defined below:

$$
\mathrm{ICI}=\frac{\text { Power }_{\mathrm{ICI}}}{\text { Power }_{\text {Desired }}} .
$$

ISI is another impairment in communication systems caused by the adjacent symbols (possibly operating on different frequencies) in time mainly due to delay spread. Another important possible cause of ISI for orthogonal pulse shapes overlapping in time is fast fading channels where the orthogonality of the adjacent pulse shapes may be broken due to different channel complex gain per sample within a single impulse duration. In this report, ISI will be referencing the ratio defined below:

$$
\text { ISI }=\frac{\text { Power }_{\text {ISI }}}{\text { Power }_{\text {Desired }}} .
$$

A combined term ISCI is defined as:

$$
\text { ISCI }=\frac{\text { Power }_{\text {ISCI }}}{\text { Power }_{\text {Desired }}}=\frac{\text { Power }_{\text {ISI }}+\text { Power }_{\text {ICI }}}{\text { Power }_{\text {Desired }}} .
$$

Therefore, ICI, ISI, and ISCI refer here to Interference to Signal Ratio. This definition of the interference term ISCI is adopted since it is the reciprocal of the Signal to Interference Ratio (SIR), fully relevant in absence of noise. The next section will use the system model built to calculate the expected received power at each time-frequency slot generated due to the transmission of a specific time-frequency slot.

\section{Interference Calculation}

\subsection{End-to-end Equation}

In this section, the end-to-end equation is to be derived by writing the power received on sub-carrier $m^{\prime}$ and $\mathrm{MC}$ symbol $n^{\prime}$ due to the input symbol modulated over sub-carrier $m$ and MC symbol $n$. Combining Equation 7, Equation 11, and Equation 13 from subsection 2.3, we can result an end-to-end equation:

$$
\begin{aligned}
\hat{c}_{m^{\prime}, n^{\prime}}= & \left\langle\boldsymbol{r}, \tilde{\boldsymbol{g}}_{m^{\prime}, n^{\prime}}\right\rangle \\
= & \left\langle\boldsymbol{h} . \boldsymbol{s}, \tilde{\boldsymbol{g}}_{m^{\prime}, n^{\prime}}\right\rangle \\
= & \left\langle\boldsymbol{h} \cdot \sum_{n} \sum_{m} \boldsymbol{s}_{m, n}, \tilde{\boldsymbol{g}}_{m^{\prime}, n^{\prime}}\right\rangle \\
= & \sum_{n} \sum_{m}\left\langle\boldsymbol{h} \cdot \boldsymbol{g}_{m, n}, \tilde{\boldsymbol{g}}_{m^{\prime}, n^{\prime}}\right\rangle c_{m, n} \\
= & \sum_{q} \sum_{n} \sum_{m} \tilde{\boldsymbol{g}}^{*}\left[q-n^{\prime} N\right] \\
& \times h[q] g[q-n N] c_{m, n} \\
& \times e^{j 2 \pi \frac{q m-q m^{\prime}-N m n+N m^{\prime} n^{\prime}}{M}} .
\end{aligned}
$$


As it can now be seen in Equation 19, the fundamental impact on the received values is due to the inner product $\left\langle\boldsymbol{h} \boldsymbol{g}_{m, n}, \tilde{\boldsymbol{g}}_{m^{\prime}, n^{\prime}}\right\rangle$, as expected from Equation 1 and illustrations and interpretations of subsection 2.2.

Introducing $\gamma=q-n N$, the range of $\gamma$ can be defined using the range of $q$ defined in Equation 15, resulting in $\gamma \in\left[n^{\prime} N ; K N-1\right]$. Substituting $\gamma$ in Equation 19:

$$
\begin{aligned}
\hat{c}_{m^{\prime}, n^{\prime}}= & \sum_{\gamma} \sum_{n} \sum_{m} \tilde{g}^{*}\left[\gamma-N\left(n^{\prime}-n\right)\right] \\
& \times h[\gamma+n N] g[\gamma] c_{m, n} \\
& \times e^{-j 2 \pi \frac{\gamma\left(m^{\prime}-m\right)-m^{\prime} N\left(n^{\prime}-n\right)}{M}} .
\end{aligned}
$$

For a given TX symbol transmitted at frequency-time indices $(m, n)$, the contribution on the RX indices $\left(m^{\prime}, n^{\prime}\right)$ are:

$$
\begin{aligned}
\left.\hat{c}_{m^{\prime}, n^{\prime}}\right|_{m, n}= & \left\langle\boldsymbol{h} \cdot \boldsymbol{g}_{m, n}, \tilde{\boldsymbol{g}}_{m^{\prime}, n^{\prime}}\right\rangle c_{m, n} \\
= & \sum_{\gamma} \tilde{g}^{*}\left[\gamma-N\left(n^{\prime}-n\right)\right] \\
& \times h[\gamma+n N] g[\gamma] c_{m, n} \\
& \times e^{-j 2 \pi \frac{\gamma\left(m^{\prime}-m\right)-m^{\prime} N\left(n^{\prime}-n\right)}{M}} .
\end{aligned}
$$

It is worth noticing that for a know $n$ as in Equation 21, the range of $\gamma$ is altered to $\gamma \in\left[n^{\prime} N ; K N-1\right]$.

\subsection{Expected Received Power}

The expected received power at symbol $\hat{c}_{m^{\prime}, n^{\prime}}$ due to the transmitted signal of input symbol $c_{m, n}$ is:

$$
\begin{gathered}
\left.P_{m^{\prime}, n^{\prime}}\right|_{m, n}=E\left[\left.\left|\hat{c}_{m^{\prime}, n^{\prime}}\right|_{m, n}\right|^{2}\right]=E\left[\left(\left.\hat{c}_{m^{\prime}, n^{\prime}}\right|_{m, n}\right)\left(\left.\hat{c}_{m^{\prime}, n^{\prime}}\right|_{m, n}\right)^{*}\right] \\
=\sigma^{2} \sum_{\gamma} \sum_{\gamma^{\prime}} E\left[\tilde{g}^{*}\left[\gamma-N\left(n^{\prime}-n\right)\right] \tilde{g}\left[\gamma^{\prime}-N\left(n^{\prime}-n\right)\right]\right. \\
\left.\times h[\gamma+n N] h^{*}\left[\gamma^{\prime}+n N\right] g[\gamma] g^{*}\left[\gamma^{\prime}\right] e^{-j 2 \pi \frac{\left(m^{\prime}-m\right)\left(\gamma-\gamma^{\prime}\right)}{M}}\right],
\end{gathered}
$$

such that $E[$.$] is the expectation operator, and \sigma^{2}=$ $E\left\{\left|c_{m, n}\right|^{2}\right\}$ is the variance of i.i.d zero-mean input symbols $c_{m, n}$. Defining $R_{h}\left(x=\gamma-\gamma^{\prime}\right)=E\left[h[\gamma] h^{*}[\gamma-x]\right]$ as the channel's complex gains auto-correlation function (invariant over $n$ ), it can be plugged into Equation 22 to get:

$$
\begin{aligned}
& \left.P_{m^{\prime}, n^{\prime}}\right|_{m, n}= \\
& =\sigma^{2} \sum_{\gamma} \sum_{\gamma^{\prime}} \tilde{g}^{*}\left[\gamma-N\left(n^{\prime}-n\right)\right] \tilde{g}\left[\gamma^{\prime}-N\left(n^{\prime}-n\right)\right] \\
& \quad \times R_{h}\left(\gamma-\gamma^{\prime}\right) g[\gamma] g^{*}\left[\gamma^{\prime}\right] e^{-j 2 \pi} \frac{\left(m^{\prime}-m\right)\left(\gamma-\gamma^{\prime}\right)}{M} .
\end{aligned}
$$

As shown in Equation 23, the received power is not dependent on the time symbol index and frequency sub-carrier index, but on the (time) offset between symbols and the (frequency) offset between sub-carriers. Using the index $\Delta=\left[\Delta_{c}, \Delta_{s}\right]$ defined in Equation 2, Equation 23 can be re-written as:

$$
\begin{aligned}
P_{\Delta}= & \sigma^{2} \sum_{\gamma} \sum_{\gamma^{\prime}} \tilde{g}^{*}\left[\gamma-N \Delta_{s}\right] \tilde{g}\left[\gamma^{\prime}-N \Delta_{s}\right] \\
& \times R_{h}\left(\gamma-\gamma^{\prime}\right) g[\gamma] g^{*}\left[\gamma^{\prime}\right] e^{-j 2 \pi \frac{\Delta_{c}\left(\gamma-\gamma^{\prime}\right)}{M}} .
\end{aligned}
$$

Following the assumption of a Jakes model Rayleigh channel, the channel auto-correlation function is then defined by the Bessel function of the first kind as $R_{h}(x)=J_{0}\left(2 \pi F_{d} T_{S a} x\right)$. In addition to that, the input symbols are assumed to have a unitary average power $\sigma^{2}=1$. Updating Equation 24 accordingly:

$$
\begin{aligned}
P_{\Delta}= & \sum_{\gamma} \sum_{\gamma^{\prime}} \tilde{g}^{*}\left[\gamma-N \Delta_{s}\right] \tilde{g}\left[\gamma^{\prime}-N \Delta_{s}\right] \\
& \times J_{0}\left(2 \pi F_{d} T_{S a}\left(\gamma-\gamma^{\prime}\right)\right) g[\gamma] g^{*}\left[\gamma^{\prime}\right] e^{-j 2 \pi \frac{\Delta_{c}\left(\gamma-\gamma^{\prime}\right)}{M}} .
\end{aligned}
$$

We observe that the above-concluded power versus $\Delta$ depends on $g, \tilde{g}^{*}, T_{S}$, and $F_{d}$ where only $F_{d}$ is a non-configurable parameter and depends on the channel's properties. The Equation 25 derived above can be used directly with normalized Doppler frequency $F_{d} T_{S}$ despite the value of each term separately for the sake of generalization. These equations are used in the next section to provide simulation results for the interference power and their analysis.

Considering the interference level after the receiving correlator, the expected power Equation 25 can be used to calculate the values of the power terms defined in subsubsection 2.3 .5 by:

$$
\text { Power }_{\text {Desired }}=P_{\Delta=\left[\begin{array}{ll}
0 & 0
\end{array}\right]},
$$

the ICI power can be obtained using the following summation:

$$
\text { Power }_{\mathrm{ICI}}=\sum_{\substack{\Delta_{s}=0 \\ \Delta_{c} \neq 0}} P_{\Delta},
$$

the ISI power can be obtained by:

$$
\text { Power }_{\text {ISI }}=\sum_{\Delta_{s} \neq 0} P_{\Delta}
$$

and the ISCI power can be obtained by:

$$
\text { Power }_{\text {ISCI }}=\text { Power }_{\text {ISI }}+\text { Power }_{\text {ICI }}
$$

So it is to note from Equation 27 and Equation 28 that ISI counts all the interference from the undesired MC symbol, whereas ICI counts only the interference between sub-carriers inside the desired MC symbol. The values of power obtained in Equation 26, Equation 27, Equation 28, and Equation 29 can be plugged in into Equation 16, Equation 17, and Equation 18 introduced in subsubsection 2.3.5 Interference Definition. 

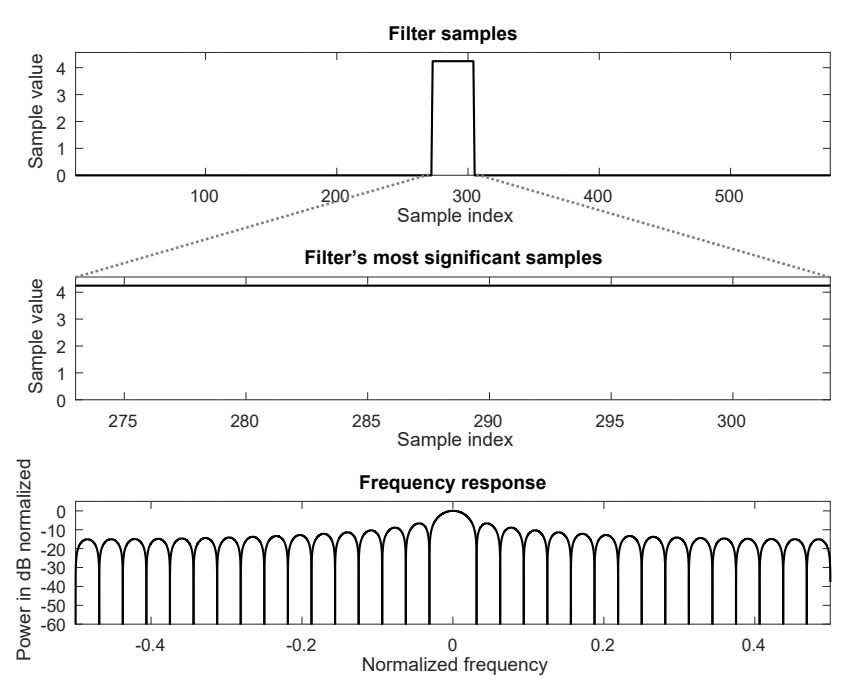

Figure 8: Rect pulse time and frequency response.

\section{Simulations and Analysis}

\subsection{Simulation Setup}

In this section, the number of MC symbol samples spacing $N=36$, the number of sub-channels $M=32$, and the pulse length in terms of MC symbols $K=15$ are used (which leads to a pulse length of $K \times N=540$ samples). The configured $M$ and $N$ results in $T_{S}=N T_{S a}$ and $F_{S}=\frac{B}{M}=\frac{N}{M T_{S}}$ for sample time $T_{S a}$, symbol time $T_{S}$, sub-carrier spacing $F_{S}$, and total MC bandwidth $B$. Monte-Carlo simulations using 512 observations are shown in scattered marker-sets signified by subscript 'MC' in legends, and results based on the concluded expected power in Equation 25 are shown in the solid-line plots. Notice that for this setup, a ratio of $N / M=1.125>1$ is used which signifies an oversampled OFDM with a subcarrier spacing $F_{S}=1.125 \times B_{S C}^{\min }$ (contrary to subsection 2.2 where $\left.F_{S}=B_{S C}^{\text {min }}\right)$. A ratio greater than 1 (oversampled) was adopted since according to Balian-Low Theorem [29], it is not possible to get well time-frequency localized pulses while considering exact sampling or to be said differently, it is not possible to limit the pulse spreading in both time and frequency if the number of samples of the pulse equals the number of sub-carriers, whereas it will be shown later in this paper, localization significantly affects the interference level. The pulse shape at the receiver is assumed to be matched to the transmitter's pulse shape, which means that $\tilde{g}_{m, n}=g_{m, n}$. To cover as many general cases as possible, the following pulse shapes are used in the simulations:

- Rect pulse shown in Figure 8 noted as 'Rect' in legends. Used to compare with classical OFDM.

- RRC pulse shown in Figure 9 noted as ' $\mathrm{RRC}^{\alpha}$ ' in legends where $\alpha$ is the roll-off factor. Used to compare with the band-limited orthogonal pulse shapes family. Two values of roll-off $\alpha$ are considered: 0.125 and 1 . Roll-off $\alpha=0.125$ is used since it was found to have minimal ISCI for a static channel using the considered
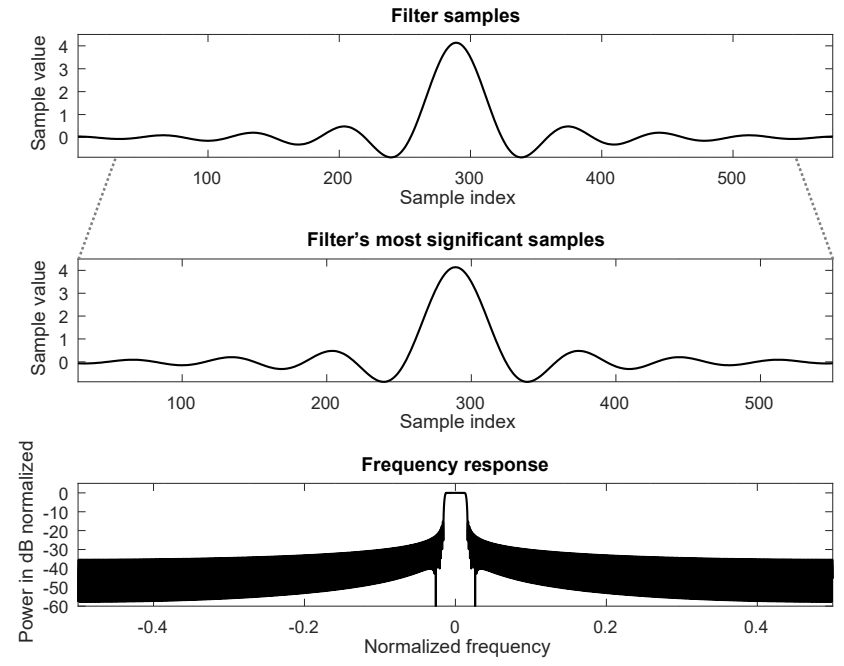

Figure 9: RRC pulse time and frequency response.

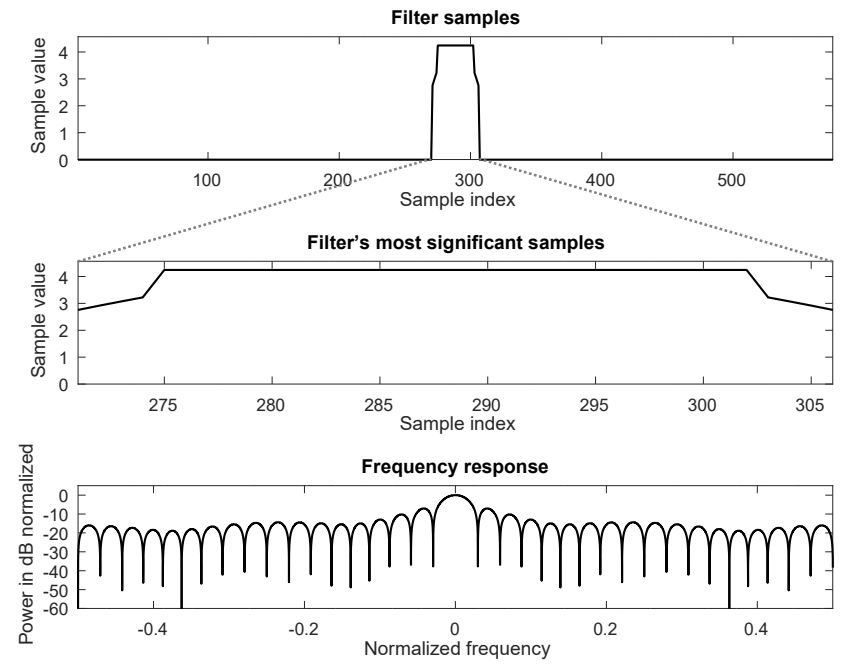

Figure 10: OBE pulse time and frequency response.

$M, N$, and $K$ because it is the maximum possible $\alpha$ that would introduce no ICI for a static channel. Rolloff $\alpha=1$ is used as an extreme case that is expected to minimizes the ISI thanks to the fast decay in time domain and then better time-localisation (as ISI is a major problem to RRC pulse shapes as shown in subsection 2.2). The RRC pulse sample was generated by taking samples at $t=k T_{S a} k \in \mathbb{Z}$ using the Equation 30 below extracted from reference [30]:

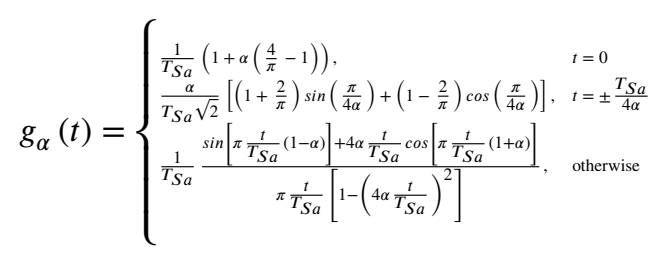

- OBE pulse shown in Figure 10 noted as 'OBE' in legends. Used to compare with the time-limited orthogo- 


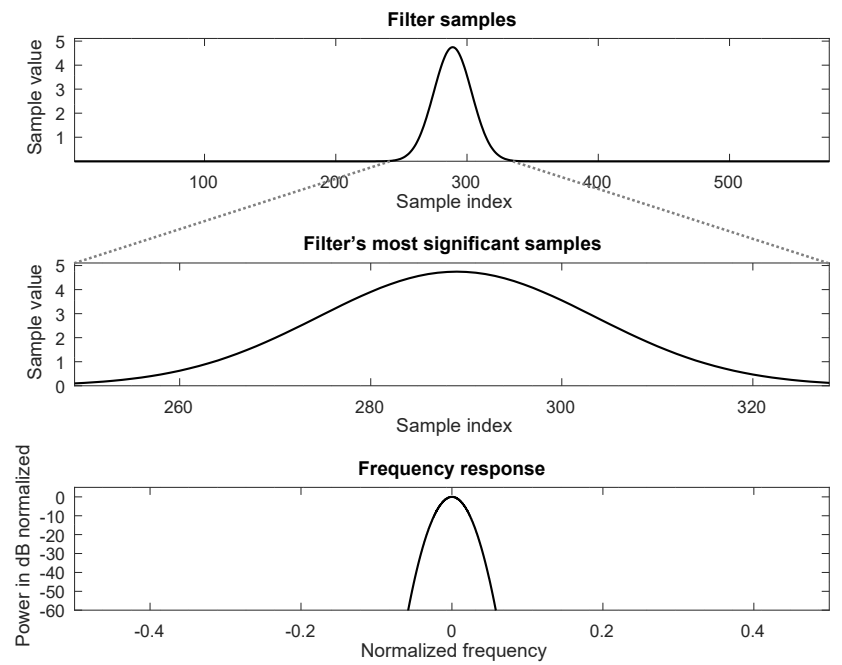

Figure 11: Gauss pulse time and frequency response.

nal pulse shapes family as it is shown in reference [31] to have minimal out of band emissions as a time-limited pulse.

- Gauss pulse shown in Figure 11 noted as 'Gauss' in legends. Used to compare with the non-orthogonal pulse shapes family, yet semi-limited in both time and frequency domain. Gauss pulse of variance $208 T_{S a}^{2}$ for a sample time $T_{S a}$ is used in this paper. This variance was used since it was found to have minimal ISCI and nearly equal ISI and ICI for a slow-varying channel $\left(F_{d} T_{S} \approx 0\right)$ using the considered $M, N$, and $K$ by performing brute-force simulations using the Gaussian equation for variance $\alpha^{2}$ and time instance $t=k T_{S a} \quad k \in \mathbb{Z}$ :

$$
g_{\alpha}(t)=\frac{1}{\sqrt{2 \pi \alpha}} e^{\frac{-t^{2}}{2 \alpha}} .
$$

\subsection{Interference versus Doppler spread}

In Figure 12, a plot of ISCI versus Doppler spread $F_{d} T_{S}$ is shown. First of all, we can see in this figure that the analytic formula perfectly matches with simulation results. These observation stands for all the next figures and will not be repeated. For low Doppler spread, it is clear how Gauss pulse shape generates the most ISCI due to its non-orthogonal nature even for no Doppler spread. For the RRC pulse shape, although it is theoretically orthogonal, as a band-limited pulse shape it has an unlimited impulse response that cannot be realized. Consequently, for $\mathrm{RRC}^{1 / 8}$ pulse, it generates slight interference even for no Doppler spread due to the orthogonality broken by the truncation, while for $\mathrm{RRC}^{1}$, most of the interference is generated due to the maximum roll-off factor. Such an effect for high roll-off is because of having a pulse shape bandwidth of $\frac{2}{T_{S}}$ while the sub-carrier spacing is $F_{S}=\frac{N}{M T_{S}}=\frac{1.125}{T_{S}}$. Time-limited pulse shapes like $\mathrm{OBE}$ and Rect, which can be easily realized, have no interference for no Doppler spread. For all pulse shapes, ISCI

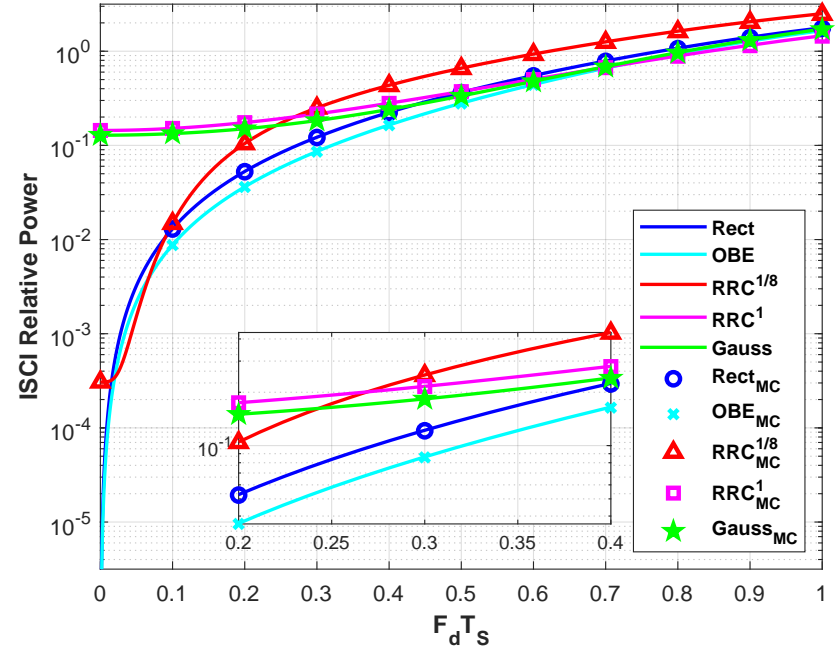

Figure 12: ISCl versus $F_{d} T_{S}$ for different pulse shapes.

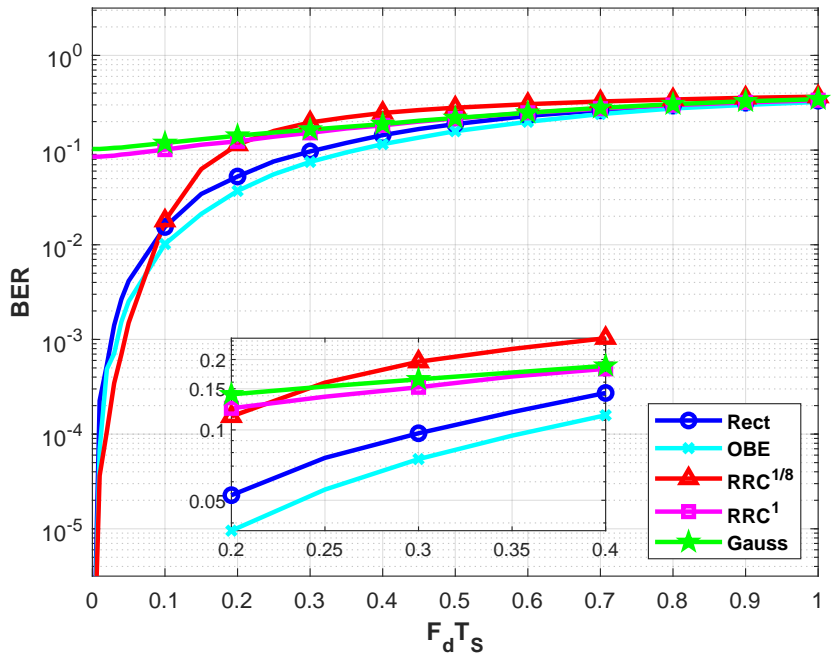

Figure 13: Bit Error Rate (BER) versus $F_{d} T_{S}$ for different pulse shapes for QAM16 constellation.

increases as Doppler spread increases as expected, however, $\mathrm{RRC}^{1 / 8}$ pulse shape is the most affected one, then Rect and OBE lower than $\mathrm{RRC}^{1 / 8}$ with OBE slightly better, and Gauss pulse shape is the least affected by the Doppler spread since there is no orthogonality to be broken, and $\mathrm{RRC}^{1}$ since it is limited in both time and frequency domain with interference accepted in the frequency domain.

In Figure 13, BER values versus $F_{d} T_{S}$ are provided by simulation of 10000 FDM symbols with $M=32, N=36$ and $K=15$ having i.i.d QAM16 symbols as input. In contrary to [32] that uses a sophisticated interference cancellation receiver in presence of noise, Zero Forcing equalizer with perfect channel state information, and without adding noise $(\mathrm{SNR}=\infty)$ is considered. This setup is assumed since the concern of the BER analysis provided is the impact of the interference on BER without considering any other factors. The impact of the value of $F_{d} T_{S}$ on the BER values appeared to be similar to the impact on the values of ISCI as the curves 


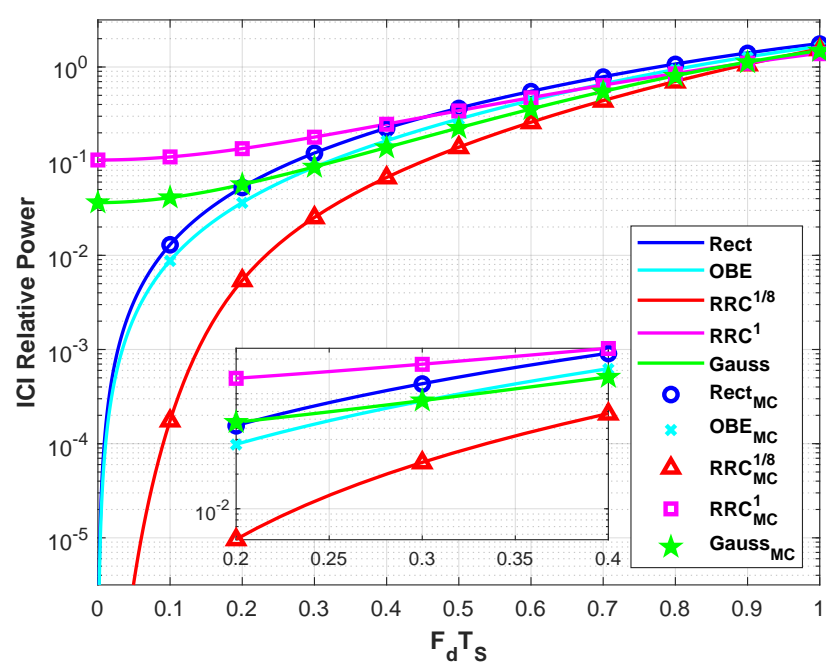

Figure 14: ICl versus $F_{d} T_{S}$ for different pulse shapes.

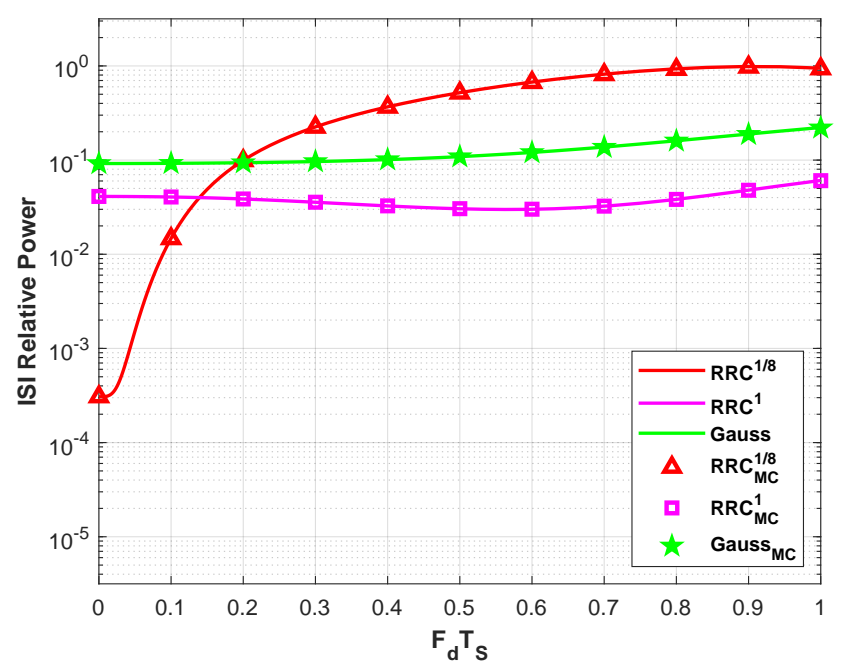

Figure 15: ISI versus $F_{d} T_{S}$ for different pulse shapes.

in Figure 12 and Figure 13.

Although the main concern due to Doppler spread is ICI, which is expected to be the lowest in band-limited pulse shapes, it is shown that OBE, which is a time-limited bandunlimited pulse shape, shows the best performance. Consequently, further analysis is required at ICI and ISI levels separately. Figure 14 shows the ICI versus Doppler spread. It shows how time-limited pulse shapes have the same ICI plot as ISCI plot of Figure 12 due to the no time-overlapping nature of these pulse shapes. As for Gauss and RRC pulse shapes, since they have ISI part, they got ICI lower than ISCI. Figure 14 shows that $\mathrm{RRC}^{1 / 8}$ pulse shape has the best performance in terms of ICI which is expected due to its band-limited nature (minimum out of band power). For the ISI versus Doppler spread shown in Figure 15, OBE and Rect have no values since as mentioned before: time-limited non-overlapping pulses do not generate ISI for single-path channels (ISI tends to 0 ). It appears how $\mathrm{RRC}^{1 / 8}$ pulse shape has significant ISI although the assumption of a single-path is

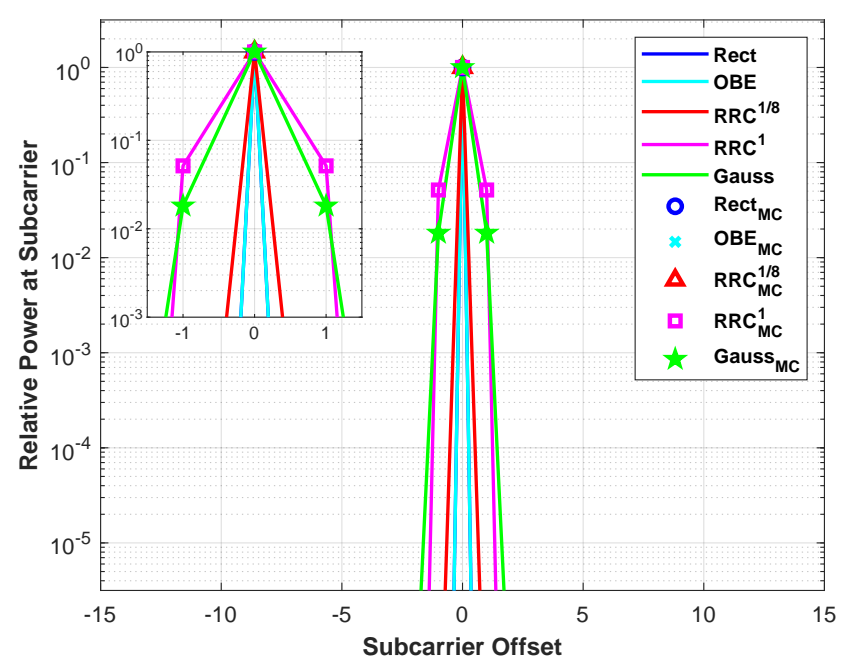

Figure 16: Received power in response to a single TX pulse versus $\mathrm{RX}$ sub-carrier offset, for $F_{d} T_{S}=0.0$, and different pulse shapes.

considered due to the orthogonality broken because of truncation. This explains the lower than expected net performance of $\mathrm{RRC}^{1 / 8}$ shown in Figure 12. For the $\mathrm{RRC}^{1}$ pulse, the high roll-off factor (1) allows significant interference, but mainly from the first two neighbors in the frequency domain. This appears in ISI due to the cross-terms (interference coming from different symbols at different sub-carrier frequencies). It can be noticed that for $\mathrm{RRC}^{1}$, in contrary to $\mathrm{RRC}^{1 / 8}$, although high ISI values exist even for low $F_{d} T_{S}$, the value does not change significantly as $F_{d} T_{S}$ increases as it can be noticed that over the full range of analyzed $F_{d} T_{S}$ values, the value of ISI is bounded by a variation of $\sim 3 \mathrm{~dB}$. This robustness of RRC ${ }^{1}$ against Doppler-generated-ISI is due to its fast decaying pulse shape in the time domain.

The general performance analyzed above can be better shown by analyzing per-symbol and per-sub-carrier interference. Therefore, in the next two sections, we will see how the activation of only one given TX pulse (a single sub-carrier transmitted at one symbol MC instant) can produce instantaneous received power in the output of the bank of the RX pulse shapes (each analyzing different sub-carrier index), and for different time symbol MC index.

\subsection{Interference versus sub-carrier offset}

Figure 16 and Figure 17 show the power received per subcarrier offset per pulse shape for $F_{d} T_{S}=0.0$ and $F_{d} T_{S}=0.2$ respectively (in other words, the term $P_{\Delta_{c}}$ of Equation 27 is plotted versus $\Delta_{c}$ ). These plots show that Rect and OBE pulse shapes have higher ICI than $\mathrm{RRC}^{1 / 8}$, as it was shown previously in Figure 14. Again, as expected, $\mathrm{RRC}^{1 / 8}$ is significantly affecting and affected by only its nearest two neighbors. For the Gauss pulse shape, since it is semi-limited in both frequency and time domain, although it has higher total interference than Rect and OBE, most of the interference power is on the nearest two neighbors and slight interference on the second nearest two neighbors. $\mathrm{RRC}^{1}$ has similar behavior to Gauss in terms of ICI due to the high roll-off. This 


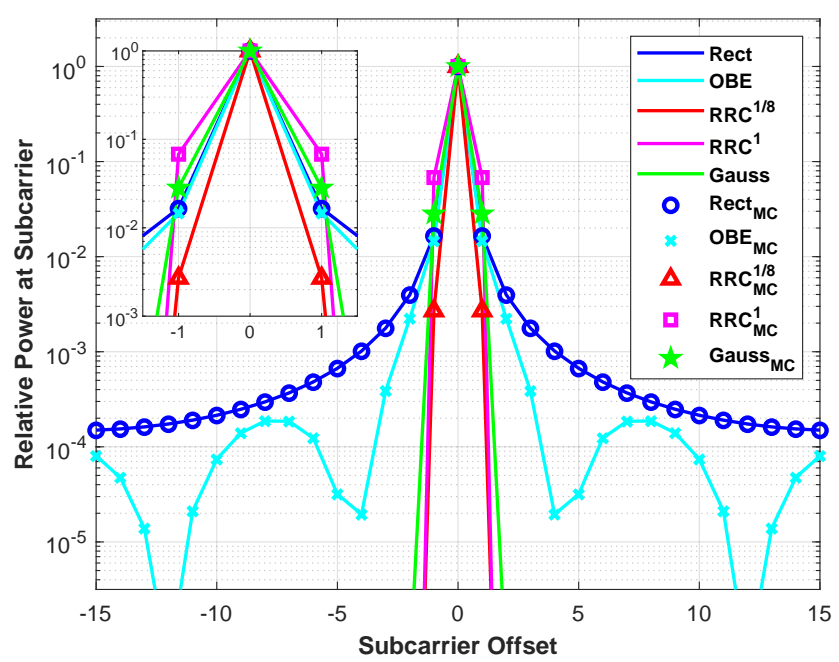

Figure 17: Received power in response to a single TX pulse versus RX sub-carrier offset, for $F_{d} T_{S}=0.2$, and different pulse shapes.

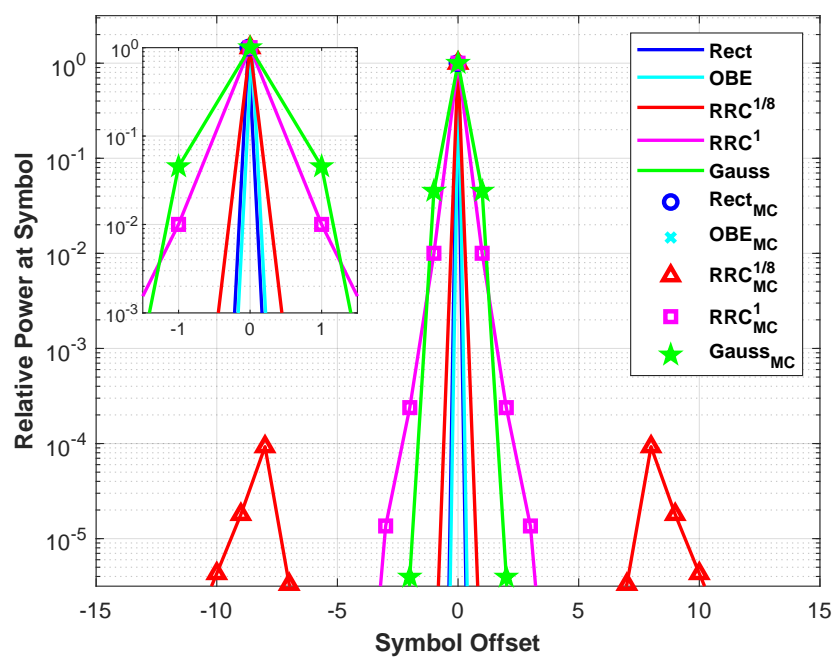

Figure 18: Received power in response to a single TX pulse versus $\mathrm{RX}$ symbol offset, for $F_{d} T_{S}=0.0$, and different pulse shapes.

high roll-off for $\mathrm{RRC}^{1}$ limits the 'tail' of the pulse improving its time-localization while maintaining fine frequency localization by spreading at only twice the minimum bandwidth. This relatively good localization comes at the cost of accepting ICI from the first two neighbor sub-carriers.

\subsection{Interference versus symbol offset}

Figure 18 and Figure 19 show the power received per symbol offset per pulse shape for $F_{d} T_{S}=0.0$ and $F_{d} T_{S}=0.2$ respectively (in other words, the term $P_{\Delta_{s}}$ of Equation 17 is plotted versus $\Delta_{S}$ ). These plots show that time-limited pulse shapes Rect and OBE have no ISI for single-path channel even with Doppler spread, as it was shown previously in Figure 15 , while $\mathrm{RRC}^{1 / 8}$ has increased ISI due to further broken orthogonality. For the Gauss pulse shape, since it is semilimited in both frequency and time domain, it has higher total

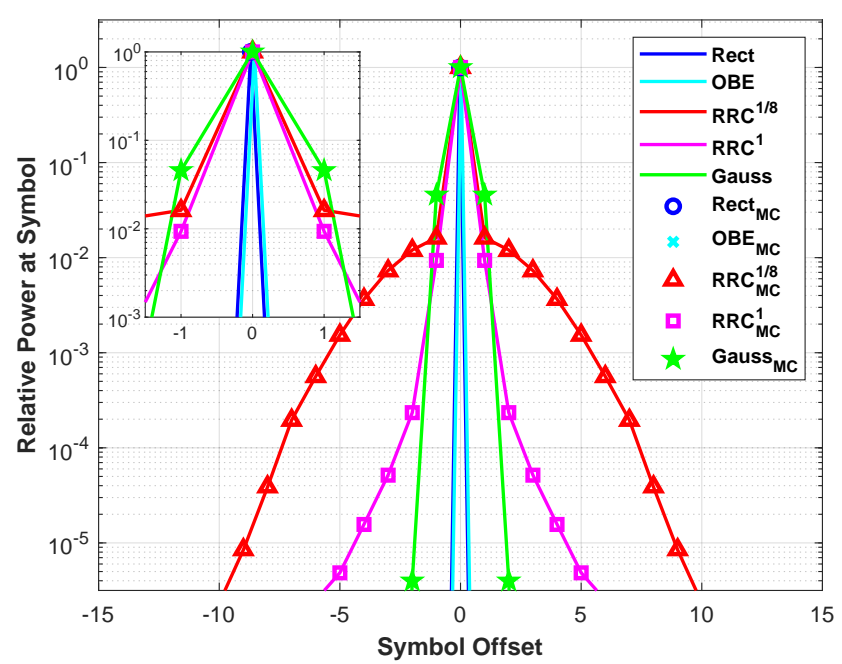

Figure 19: Received power in response to a single TX pulse versus $\mathrm{RX}$ symbol offset, for $F_{d} T_{S}=0.2$, and different pulse shapes.

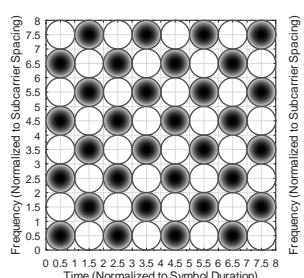

(a)

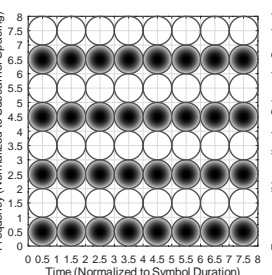

(b)

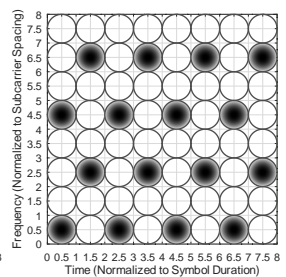

(c)
Figure 20: Symbols distribution over the symbol-offset/subcarrier-offset grid for a) $(m+n)=2 k$, b) $m=2 k$, and c) $(m+2 n)=4 k$ all for $k \in \mathbb{Z}$, black is active, and white is disabled.

interference than $\mathrm{RRC}^{1 / 8}$, but most of the interference power is on the nearest two neighbors and the rest on the second nearest two neighbors. For the RRC ${ }^{1}$ pulse, most of the ISI power is due to the cross-terms (from different sub-carrier of different symbols) as mentioned before.

\subsection{Reducing FDM Symbol Density}

In subsection 4.3 and subsection 4.4, it appeared how $\mathrm{RRC}^{1}$ pulse shape has its interference centered to nearest two neighbors in the frequency domain, and Gauss pulse shape has its interference centered to nearest two neighbors, and slightly to the next neighbors in both frequency and time. Due to these properties, it is interesting to check the performance after reducing the symbol grid density to $50 \%$ by activating symbols only having $(m+n)=2 k$ as in Figure 20(a) resulting in Figure 21 and having $m=2 k$ as in Figure 20(b) resulting in Figure 22 both for $k \in \mathbb{Z}$. More analysis can be done after reducing the grid density to $25 \%$ by activating symbols only for $(m+2 n)=4 k$ as in Figure 20(c) resulting in Figure 23 for $k \in \mathbb{Z}$. Based on the previous discussion on the properties of the Gauss pulse shape, and the variance configuration adjusted to have even distribution in terms of ISI and ICI, it is clearly shown in Figure 21 that Gauss pulse shape is the one that benefited 


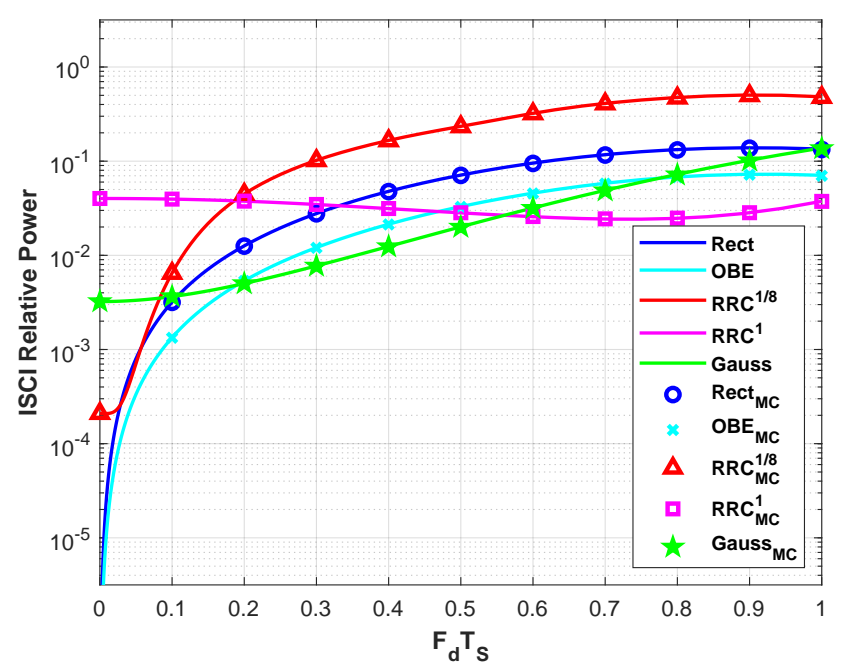

Figure 21: ISCl versus $F_{d} T_{S}$ for different pulse shapes for $50 \%$ density with $(m+n)=2 k$ for $k \in \mathbb{Z}$.

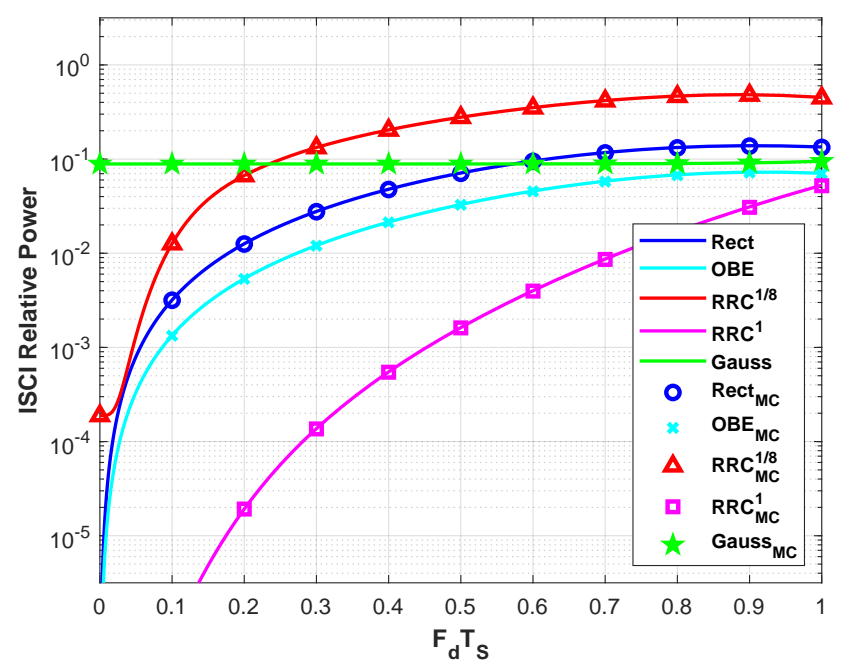

Figure 22: $\mathrm{ISCl}$ versus $F_{d} T_{S}$ for different pulse shapes for $50 \%$ density with $m=2 k$ for $k \in \mathbb{Z}$.

the most from the symbol density reduction with the grid in Figure 20(a) due to maximizing the time-frequency distance between symbols and making it even. However, the $\mathrm{RRC}^{1}$ pulse shape: 1) although introducing interference, has a good localization in frequency domain due to limited-fastdecaying transfer function, 2) has a good time localization due to fast decaying caused by high roll-off which helped in making the orthogonality to the adjacent symbols not broken easily, and 3) has most of its interference power coming from ICI (even in ISI it comes mostly from $m^{\prime} \neq m \& n^{\prime} \neq n$ ). Due to such properties, it appeared that $\mathrm{RRC}^{1}$ requires more protection from adjacent frequencies what resulted in having the lowest interference power in Figure 22 when using the grid in Figure 20(b). For example, in [5], the authors have successfully experimented with wireless transmission at carrier frequency $0.325 \mathrm{THz}$ using 64-QAM-OFDM without considering the receiver's movement. Considering this carrier frequency, and using the standard Long-Term Evolution

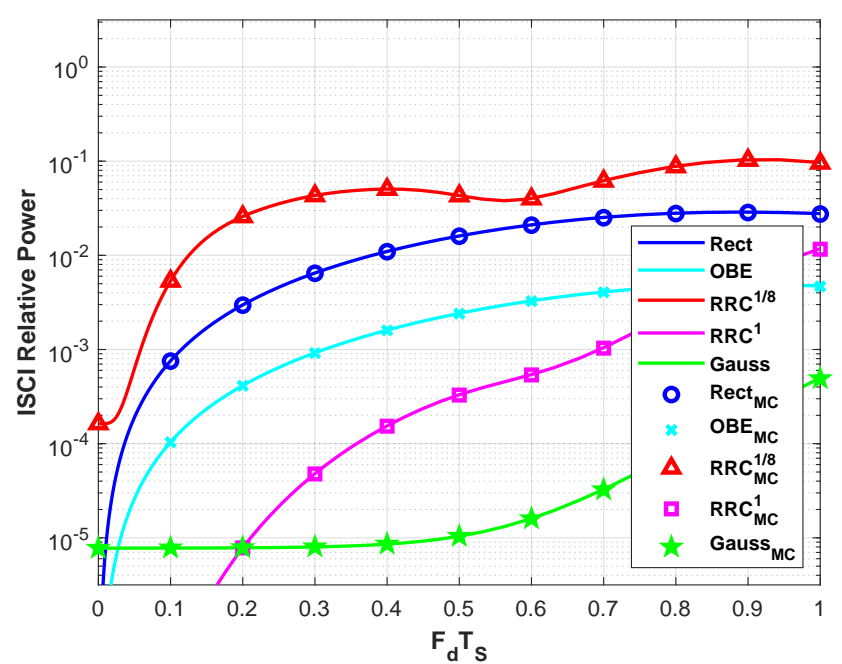

Figure 23: ISCl versus $F_{d} T_{S}$ for different pulse shapes for $25 \%$ density with $(m+2 n)=4 k$ for $k \in \mathbb{Z}$.

(LTE) sub-carrier frequency spacing $15 \mathrm{kHz}$, a person walking (or running) at speed of $3 \mathrm{~m} / \mathrm{s}$ will experiment a Doppler frequency $F_{d}=\frac{3}{3 \times 10^{8}} \times 0.325 \times 10^{12}=3.25 \mathrm{kHz}$ and have a normalized Doppler spread $F_{d} T_{S} \approx 0.2$. Using normal OFDM will have $\mathrm{SIR} \approx 16 \mathrm{~dB}$, even when reducing the grid density to $50 \%$, an SIR $\approx 19.5 \mathrm{~dB}$ is obtained. However, when switching to $\mathrm{RRC}^{1}$, an SIR $\approx 49 \mathrm{~dB}$ is obtained. In such a scenario, using $\mathrm{RRC}^{1}$ will enhance the performance by $\sim 30 \mathrm{~dB}$ compared to standard OFDM and by $\sim 23 \mathrm{~dB}$ compared to the best alternative in the simulated pulse shapes, allowing to use higher order of constellation. In Figure 22, it is shown that even for the RRC ${ }^{1}$ that appeared to have a very good performance in the mid-range $F_{d} T_{S}$ with $m=2 k$, but interference levels became high again at high range $F_{d} T_{S}$. Performing further density reduction to reach $25 \%$ appeared to be not very beneficial for $\mathrm{RRC}^{1}$, however, it shows how the Gauss pulse shape benefited significantly, producing an acceptable value of interference over all the range of simulation. As it was expected, the pulse shapes having the least interference spreading benefited the most from density reduction, as most of the interference is canceled out by reducing the density. Due to the performance of Gauss pulse shape, the next section will illustrate and analyze further the special ability of the Gauss pulse shape to have arbitrary offset in both domains, time and frequency, with alignments constraints.

\section{Soft-Spacing Interference Calculation}

As shown in subsection 4.5 increasing the spacing (in both / either time and / or frequency) changes the performance of the pulse shapes differently, and as a consequence, changes the selection of the most adequate pulse shape in terms of interference reduction. For more dynamic analysis, this section introduced the new set of parameters $\Lambda=\left[\lambda_{c}, \lambda_{s}\right]$, where $\lambda_{c}$ is the sub-carrier spacing factor and $\lambda_{s}$ is the symbol spacing factor. The spacing increase or decrease depending on $\Lambda$ is illustrated in Figure 24 


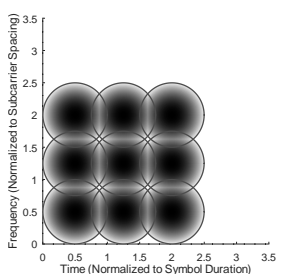

(a)

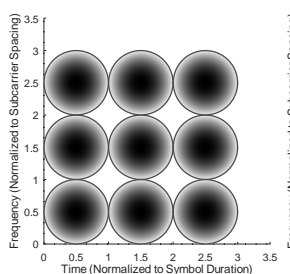

(b)

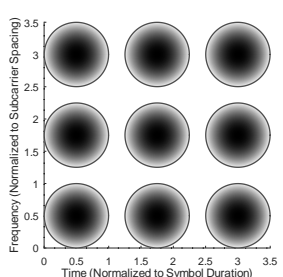

(c)
Figure 24: Symbols distribution over the symbol-offset/subcarrier-offset grid for a) $\Lambda=\left[\begin{array}{ll}0.75 & 0.75\end{array}\right]$, b) $\Lambda=$ [1.00 1.00], and c) $\Lambda=\left[\begin{array}{ll}1.25 & 1.25\end{array}\right]$.

\subsection{Soft-Spacing ISCI Re-derivation}

Injecting the just introduced $\Lambda$ into the Equation 9 and Equation 12, the updated pulse shapes will respectively be:

$$
\begin{aligned}
& g_{m, n, \Lambda}[q]=g\left[q-n \lambda_{s} N\right] e^{j 2 \pi \frac{\lambda_{c} b\left(q-n \lambda_{S} N\right)}{M}} \text {, and } \\
& \tilde{g}_{m^{\prime}, n^{\prime}, \Lambda}^{*}[q]=\tilde{g}^{*}\left[q-n^{\prime} \lambda_{S} N\right] e^{-j 2 \pi \frac{\lambda_{c} p\left(q-n^{\prime} \lambda_{s} N\right)}{M}} .
\end{aligned}
$$

Similar to Equation 21 derivation, the received symbol using pulse shapes updated in Equation 32 and Equation 33, and $\gamma=q-n \lambda_{s} N$ :

$$
\begin{aligned}
\left.\hat{c}_{m^{\prime}, n^{\prime}, \Lambda}\right|_{m, n}= & \sum_{\gamma} \tilde{g}^{*}\left[\gamma-\lambda_{s} N\left(n^{\prime}-n\right)\right] \\
& \times h\left[\gamma+n \lambda_{s} N\right] g[\gamma] c_{m, n} \\
& \times e^{-j 2 \pi \frac{\lambda_{c}\left(\gamma\left(m^{\prime}-m\right)-m^{\prime} \lambda_{s} N\left(n^{\prime}-n\right)\right)}{M}} .
\end{aligned}
$$

Introducing $N^{\prime}=\lambda_{s} N$ and $M^{\prime}=M / \lambda_{c}$. Noting that it is required to have $N^{\prime}$ as an integer since used in symbol indices, and it is recommended to have $M^{\prime}$ as an integer to allow using the whole bandwidth allowed by the used sampling rate. It is to be noted that the bandwidth covered by using $M^{\prime}$ is equal to $\lambda_{c}$ times the bandwidth when using $M$, but using $M$ uniformly spaced sub-carriers. To maintain the same bandwidth, $M / \lambda_{c}$ sub-carriers should be used. Similarly, for the time allocation, the time covered by using $N^{\prime}$ is equal to $\lambda_{s}$ times the duration when using $N$. Replacing with $N^{\prime}$ and $M^{\prime}$ in Equation 34 the end-to-end equation becomes:

$$
\begin{aligned}
\left.\hat{c}_{m^{\prime}, n^{\prime}, \Lambda}\right|_{m, n}= & \sum_{\gamma} \tilde{g}^{*}\left[\gamma-N^{\prime}\left(n^{\prime}-n\right)\right] \\
& \times h\left[\gamma+n N^{\prime}\right] g[\gamma] \\
& \times e^{-j 2 \pi \frac{\gamma\left(m^{\prime}-m\right)-m^{\prime} N^{\prime}\left(n^{\prime}-n\right)+l b}{M^{\prime}}} c_{m, n} .
\end{aligned}
$$

Similar to the derivation of the received power in eq.Equation 25 , the expected received power using the introduced $\Lambda$ is

$$
\begin{aligned}
P_{\Delta, \Lambda}= & \sigma^{2} \sum_{\gamma} \sum_{\gamma^{\prime}} \tilde{g}^{*}\left[\gamma-N^{\prime} \Delta_{s}\right] \tilde{g}\left[\gamma^{\prime}-N^{\prime} \Delta_{s}\right] \\
& \times J_{0}\left(2 \pi F_{d} T_{S a}\left(\gamma-\gamma^{\prime}\right)\right) g[\gamma] g^{*}\left[\gamma^{\prime}\right] e^{-j 2 \pi \frac{\Delta_{c}\left(\gamma-\gamma^{\prime}\right)}{M^{\prime}}} .
\end{aligned}
$$

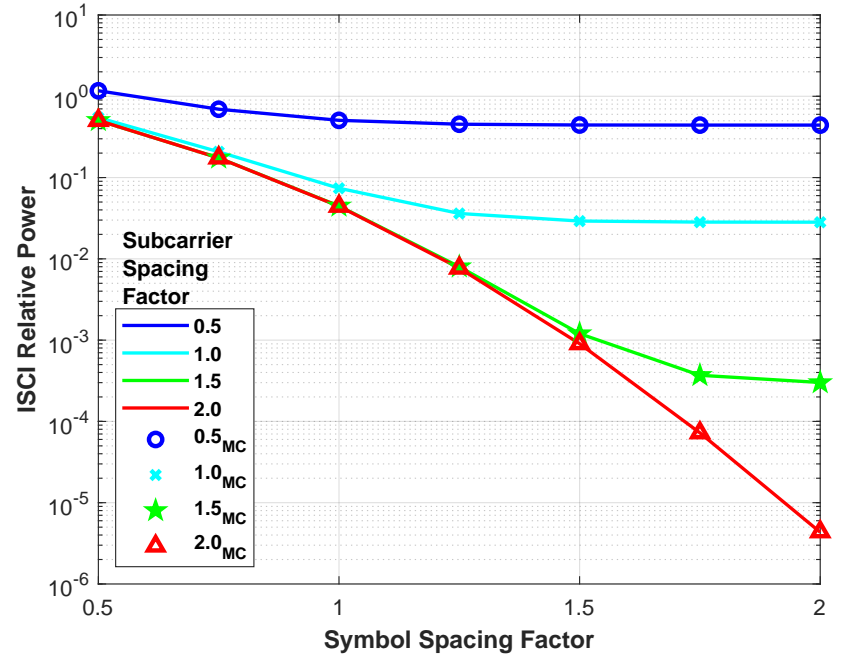

Figure 25: ISCl versus Sub-carrier frequency offset factor $\left(\lambda_{c}\right)$ per Symbol offset factor $\left(\lambda_{s}\right)$ for a Gaussian pulse shape of variance $208 T_{S a}^{2}$, for $F_{d} T_{S}=0.2$.

\subsection{Soft-Spacing ISCI results}

As expected, the plot in Figure 25 shows how the increase of the offset will induce an ISCI reduction. It can be noticed that for every value of $\lambda_{s}$, there exists a threshold of $\lambda_{c}$ that when reached, no further improvement in ISCI can be noticed (negligible). Since the frequency response of a Gaussian pulse is of Gaussian shape too, and due to the variance selected, power is distributed between time and frequency nearly evenly. Consequently, it can be seen that the threshold mentioned above is approximately when $\lambda_{s}=\lambda_{c}$. Notice that as shown in Figure 25, the variables $\lambda_{s}$ and $\lambda_{c}$ can be used to either decrease or increase the time-frequency density of the system where having the oversampling ratio $\mathrm{OR}=\frac{M^{\prime}}{N^{\prime}}=\frac{M}{N \lambda_{s} \lambda_{c}}$ such that having $\mathrm{OR}=1$ will lead to a critically sampled system, OR $>1$ will lead to an overcritical system, and OR $<1$ will lead to an undercritical system or what can be known as Faster-Than-Nyquist system as in [33].

\section{Conclusion}

In this paper, the derived end-to-end transmitted-received symbols equation and expected received power per transmitted and received symbol are used to calculate the level of interference (ISCI) in MC systems for different pulse shapebanks, Doppler spread values and the (introduced) spacing factors. It appears that different pulse shape families operate differently for different setups. It was shown that, as was expected, non-time-overlapping pulses do not generate ISI in single path channels, while they exhibit significant ICI. It was also shown that the main concern of the timeoverlapping pulses, even if designed to be orthogonal, is ISI in a fast varying channel due to changing pulse's properties. Turning off sub-carriers or equivalently expanding the symbol/carrier spacing was checked for the three discussed families and compared with OFDM. The special ability of the non-orthogonal pulse shapes of having smooth spacing is 
then discussed showing how ISCI is always decreasing with spacing increase allowing flexibility of tuning the parameters of the waveform to achieve the required maximum interference level. It was also shown that for low Doppler spreads, orthogonal pulse shapes can be used at full density. At relatively higher Doppler spread values, decreasing the system's density by $50 \%$ and using a frequency-limited pulse shape with a relatively short duration like $\mathrm{RRC}^{1}$ will outcome in the best performance in terms of interference. Furthermore, it was shown that for an extreme level of variation, the pulse shape's orthogonality will no longer be a point of interest as it will be broken due to the channel's variation. Thus, the more critical property of the pulse shape will be the localization of the interference, and a non-orthogonal fast-decaying pulse shape in both frequency and time domain like the Gauss pulse shape with further reduced density will be a better option. Although this work did not take into consideration the more general case of multi-path, which can be an extension to this work, it is still a point of interest to the domains mentioned in the introduction (multiple access [2], broadcasting [3], satellite communication [4], and terahertz communications [5]). Moreover, this work provided a deeper look into the direct effects of a very high Doppler spread on Multi-Carrier systems away from other impairments (multi-path, noise, ...).

\section{References}

[1] I Emre Telatar and David N. C. Tse. Capacity and mutual information of wideband multipath fading channels. IEEE transactions on information theory, 46(4):1384-1400, 2000.

[2] Cheong Yui Wong, Roger S Cheng, K Ben Lataief, and Ross D Murch. Multiuser OFDM with adaptive subcarrier, bit, and power allocation. IEEE Journal on selected areas in communications, 17(10):1747-1758, 1999.

[3] Hikmet Sari, Y Levy, and G Karam. An analysis of orthogonal frequency-division multiple access. In GLOBECOM 97. IEEE Global Telecommunications Conference. Conference Record, volume 3, pages 1635-1639. IEEE, 1997.

[4] Ludong Wang and Brian Jezek. OFDM modulation schemes for military satellite communications. In MILCOM 2008-2008 IEEE Military Communications Conference, pages 1-7. IEEE, 2008.

[5] Maria Freire Hermelo, Po-Tsung Boris Shih, Matthias Steeg, Anthony Ng'oma, and Andreas Stöhr. Spectral efficient 64-QAM-OFDM terahertz communication link. Optics express, 25(16):19360-19370, 2017.

[6] Eric Pierre Simon, Laurent Ros, Hussein Hijazi, Jin Fang, Davy Paul Gaillot, and Marion Berbineau. Joint carrier frequency offset and fast time-varying channel estimation for MIMO-OFDM systems. IEEE Transactions on Vehicular Technology, 60(3):955-965, 2011.

[7] Youssef Nasser, Mathieu Des Noes, Laurent Ros, and Geneviève Jourdain. On the system level prediction of joint time frequency spreading systems with carrier phase noise. IEEE transactions on communications, 58(3):839-850, 2010.

[8] Simon Bicais and Jean-Baptiste Dore. Phase noise model selection for sub-THz communications. In 2019 IEEE Global Communications Conference (GLOBECOM), pages 1-6. IEEE, 2019.

[9] Mark Russell and Gordon L Stuber. Interchannel interference analysis of OFDM in a mobile environment. In 1995 IEEE 45th Vehicular Technology Conference. Countdown to the Wireless Twenty-First Century, volume 2, pages 820-824. IEEE, 1995.

[10] Ye Li and Leonard J Cimini. Bounds on the interchannel interference of OFDM in time-varying impairments. IEEE Transactions on Communications, 49(3):401-404, 2001.
[11] Richard Hedley Clarke. A statistical theory of mobile-radio reception. Bell system technical journal, 47(6):957-1000, 1968.

[12] Behrouz Farhang-Boroujeny. OFDM versus filter bank multicarrier. IEEE signal processing magazine, 28(3):92-112, 2011.

[13] Cyrille Siclet and Pierre Siohan. Design of BFDM/OQAM systems based on biorthogonal modulated filter banks. In Globecom'O0-IEEE. Global Telecommunications Conference. Conference Record (Cat. No. OOCH37137), volume 2, pages 701-705. IEEE, 2000.

[14] Lei Zhang, Pei Xiao, Adnan Zafar, Atta ul Quddus, and Rahim Tafazolli. Fbmc system: An insight into doubly dispersive channel impact. IEEE Transactions on Vehicular Technology, 66(5):3942-3956, 2016.

[15] Dieter Schafhuber, Gerald Matz, and Franz Hlawatsch. Pulse-shaping ofdm/bfdm systems for time-varying channels: Isi/ici analysis, optimal pulse design, and efficient implementation. In The 13th IEEE International Symposium on Personal, Indoor and Mobile Radio Communications, volume 3, pages 1012-1016. IEEE, 2002.

[16] Imene Trigui, Mohammed Siala, Sofiene Affes, Alex Stephenne, and Hatem Boujemaa. Optimum pulse shaping for OFDM/BFDM systems operating in time varying multi-path channels. In IEEE GLOBECOM 2007-IEEE Global Telecommunications Conference, pages 3817-3821. IEEE, 2007.

[17] Hussein Hijazi and Laurent Ros. Joint data QR-detection and kalman estimation for OFDM time-varying rayleigh channel complex gains. IEEE Transactions on Communications, 58(1):170-178, 2010.

[18] Ali Houssam El Husseini, Eric Pierre Simon, and Laurent Ros. Secondorder autoregressive model-based kalman filter for the estimation of a slow fading channel described by the clarke model: Optimal tuning and interpretation. Digital Signal Processing, 90:125-141, 2019.

[19] A Kaye and D George. Transmission of multiplexed PAM signals over multiple channel and diversity systems. IEEE Transactions on Communication Technology, 18(5):520-526, 1970.

[20] William CY Lee. Overview of cellular CDMA. IEEE Transactions on Vehicular Technology, 40(2):291-302, 1991.

[21] Laurent Ros, Geneviève Jourdain, and Marylin Arndt. Interpretations and performances of linear reception in downlink TD-CDMA and multi-sensor extension. Annales des télécommunications, 56(5):275290, 2001.

[22] J.G. Proakis and M. Salehi. Digital Communications, 5th edition, chapter 9.2-1, pages 605-609. McGraw-Hill Higher Education, 2008.

[23] P Bello and B Nelin. Optimization of subchannel data rate in FDMSSB transmission over selectively fading media. IEEE Transactions on Communications Systems, 12(1):46-53, 1964.

[24] Uzi Timor. Equivalance of time-multiplexed and frequencymultiplexed signals in digital communications. IEEE Transactions on Communications, 20(3):435-438, 1972.

[25] Robert W Chang. Synthesis of band-limited orthogonal signals for multichannel data transmission. Bell System Technical Journal, 45 (10):1775-1796, 1966.

[26] S Weinstein and Paul Ebert. Data transmission by frequency-division multiplexing using the discrete fourier transform. IEEE transactions on Communication Technology, 19(5):628-634, 1971.

[27] Jorge Luis Seoane, Sarah Kate Wilson, and Saul Gelfand. Analysis of intertone and interblock interference in OFDM when the length of the cyclic prefix is shorter than the length of the impulse response of the channel. In GLOBECOM 97. IEEE Global Telecommunications Conference. Conference Record, volume 1, pages 32-36. IEEE, 1997.

[28] Botaro Hirosaki. An orthogonally multiplexed QAM system using the discrete fourier transform. IEEE Transactions on Communications, 29 (7):982-989, 1981.

[29] Guy Battle. Heisenberg proof of the balian-low theorem. Letters in Mathematical Physics, 15(2):175-177, 1988.

[30] Steredenn Daumont, Basel Rihawi, and Yves Lout. Root-raised cosine filter influences on PAPR distribution of single carrier signals. In 2008 3 rd International Symposium on Communications, Control and Signal Processing, pages 841-845. IEEE, 2008.

[31] Didier Pinchon and Pierre Siohan. Closed-form expressions of optimal short PR FMT prototype filters. In 2011 IEEE Global Telecommunications Conference-GLOBECOM 2011, pages 1-5. IEEE, 2011. 


\section{Robustness to Doppler in Fast Varying Flat Fading Channels}

[32] Kasturi Vasudevan, Krishnamurthy Giridhar, and Bhaskar Ramamurthi. An efficient suboptimum detector based on linear prediction in rayleigh flat-fading channels. Signal processing, 81(4):819-828, 2001.

[33] James E Mazo. Faster-than-nyquist signaling. The Bell System Technical Journal, 54(8):1451-1462, 1975.

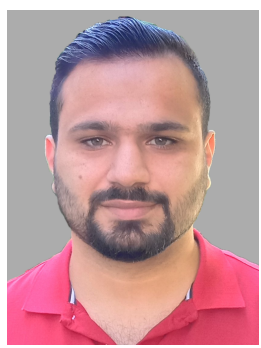

Ahmad Hamdan received the B.S. degree in science in communications engineering in 2017 and the M.S. degree in computer and communication engineering in 2019 from Lebanese International University, Beyrouth, Lebanon. He is currently working toward a Ph.D. degree in telecommunications in Université Grenoble Alpes (UGA), Grenoble, France. His thesis is concerned with high-speed radio transmission at high mobility using advanced multi-carrier modulations. His current research interests lie in the areas of signal processing and communications, including multi-carrier modulations, and channel estimation and equalization for multicarrier wireless communications.

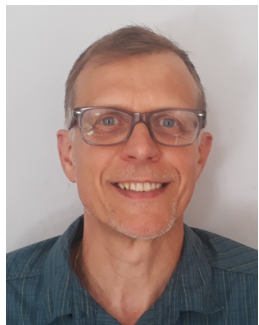

Laurent Ros received a degree in electrical engineering from Supelec, Paris, France, in 1992, and the Ph.D. and H.D.R. (accreditation to supervise research) degrees in signal processing and telecoms from the University of Grenoble, in 2001 and 2016, respectively. From 1993 to 1995 , he worked on the transmission for submarine applications with France-Telecom Research and Development, Lannion. From 1995 to 1999, he worked as a Research and Development Project Leader with Sodielec, Millau, on the design of digital modems and audio codecs for telecom equipments. Since 2002, he joins the Gipsa-laboratory (ex "Laboratory-ofImage-and-Signal") and Grenoble-INP Institute of Engineering as associate professor and then full professor in 2020. His research interest includes signal processing for wireless communications.

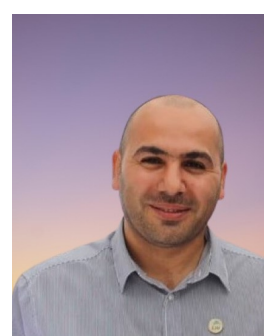

Hussein Hijazi received his Ph.D. degree in signal processing and communications from the Institut National Polytechnique de Grenoble (INPG), Grenoble, France, on 25 November 2008, where he is currently an Associate Professor. His dissertation focused on channel estimation in a high-speed mobile receiver operating in an OFDM communication system. Prior to earning his MASTER (Signal, Image, Speech, Telecom) from INPG in 2005, he was awarded the Diploma in computer and communications engineering from the Lebanese University (Faculty of Engineering), Beyrouth, Lebanon, in 2004. His current research interests lie in the areas of signal processing and communications, including synchronization, channel estimation, and equalization algorithms for wireless digital communications.

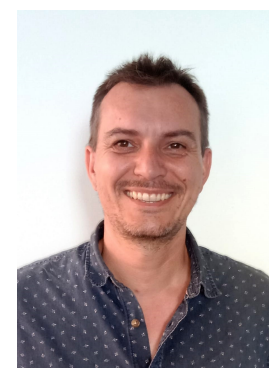

Cyrille Siclet received the engineer degree in telecommunications from the École Nationale Supérieure des Télécommunications de Bretagne (ENST Bretagne), France, in 1999 and the PhD degree in signal processing and telecommunications from the University of Rennes in 2002, respectively. He was with France Télécom R\&D, Rennes as a PhD student, from March 1999 to September 2002, with the Communications and Remote Sensing Laboratory, Université Catholique de Louvain, Louvain-la-Neuve, Belgium, from October 2002 to August 2003, as a postdoctoral researcher. $\mathrm{He}$ then worked at Université Henri Poincarré from Nancy and Centre de Recherche en Automatique de Nancy (CRAN), France, as assistant professor. Since 2004, he has been working as associate professor at GIPSA-lab (formerly known as "Laboratory of Image and Signal) and Université Grenoble Alpes (UGA, formerly known as Université Joseph Fourier) where he is involved with multicarrier systems, filterbank systems and wireless communications.

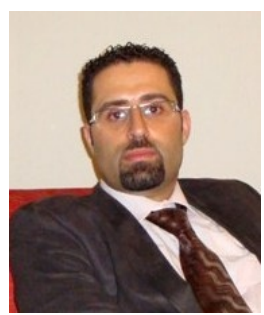

Ali Al-Ghouwayel was born in Baalbeck, Lebanon, in 1980. He received his Ph.D. degree in signalprocessing and telecommunications in 2008 from Rennes 1 University, France. His Ph.D. research activities concerned parameterization study for Software Radio systems. In 2008, he joined the LabSTICC laboratory in Lorient, France, and worked as a Postdoctoral Researcher on the FP7 European Project DAVINCI. In 2010, he joined the Lebanese International University, in Beirut-Lebanon, as Assistant Professor in the Computer and Communication Engineering department. His current researchinterests include study, optimization, and adequation algorithm-architecture for hardware implementation of non-binary LDPC decoders. 